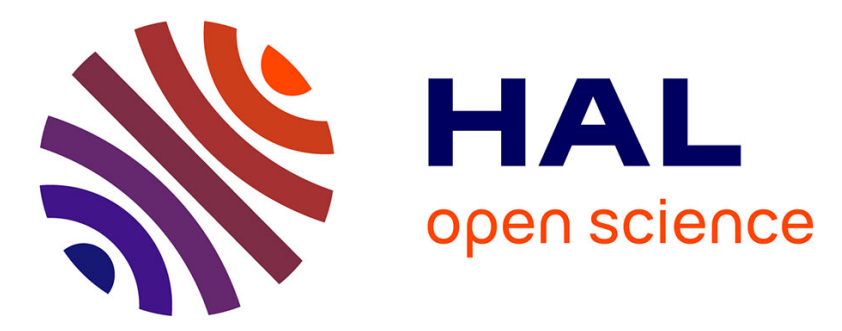

\title{
Interannual Variability in the North Atlantic Ocean's Temperature Field and Its Association with the Wind Stress Forcing
}

\author{
Andreas Groth, Yizhak Feliks, Dmitri D Kondrashov, Michael Ghil
}

\section{- To cite this version:}

Andreas Groth, Yizhak Feliks, Dmitri D Kondrashov, Michael Ghil. Interannual Variability in the North Atlantic Ocean's Temperature Field and Its Association with the Wind Stress Forcing. Journal of Climate, 2017, 30 (7), pp.2655-2678. 10.1175/jcli-d-16-0370.1 . hal-01701108

\author{
HAL Id: hal-01701108 \\ https://hal.science/hal-01701108
}

Submitted on 5 Feb 2018

HAL is a multi-disciplinary open access archive for the deposit and dissemination of scientific research documents, whether they are published or not. The documents may come from teaching and research institutions in France or abroad, or from public or private research centers.
L'archive ouverte pluridisciplinaire HAL, est destinée au dépôt et à la diffusion de documents scientifiques de niveau recherche, publiés ou non, émanant des établissements d'enseignement et de recherche français ou étrangers, des laboratoires publics ou privés. 


\title{
published in Journal of Climate, 2017, 30, 2655-2678 \\ https://doi.org/10.1175/jcli-d-16-0370.1
}

\section{Interannual variability in the North Atlantic ocean's temperature field and its association with the wind stress forcing}

\author{
Andreas Groth*, YiZHAK FELIKS ${ }^{\dagger}$, DMitri Kondrashov, AND MiChaEl GHIL ${ }^{\ddagger}$ \\ Department of Atmospheric and Oceanic Sciences, and Institute of Geophysics and Planetary Physics, University of California, Los \\ Angeles, Los Angeles, California
}

\begin{abstract}
Spectral analyses of the North Atlantic temperature field in the Simple Ocean Data Analysis (SODA) reanalysis identify prominent and statistically significant interannual oscillations along the Gulf Stream front and in large regions of the North Atlantic. A 7-8-yr oscillatory mode is characterized by a basin-wide southwest-to-northeast-oriented propagation pattern in the sea surface temperature (SST) field. This pattern is found to be linked to a seesaw in the meridional-dipole structure of the zonal wind stress forcing (TAUX). In the subpolar gyre, the SST and TAUX fields of this mode are shown to be in phase opposition, which suggests a cooling effect of the wind stress on the upper ocean layer. Over all, this mode's temperature field is characterized by a strong equivalent-barotropic component, as shown by covariations in SSTs and sea surface heights, and by phase-coherent behavior of temperature layers at depth with the SST field. Recent improvements of multivariate singular spectrum analysis (M-SSA) help separate spatio-temporal patterns. This methodology is developed further and applied to studying the ocean's response to variability in the atmospheric forcing. Statistical evidence is shown to exist for other mechanisms generating oceanic variability of similar 7-8-yr periodicity in the Gulf Stream region; the latter variability is likewise characterized by a strongly equivalent-barotropic component. Two other modes of biennial variability in the Gulf Stream region are also identified, and it is shown that interannual variability in this region cannot be explained by the ocean's response to similar variability in the atmospheric forcing alone.
\end{abstract}

\section{Introduction and motivation}

The North Atlantic Oscillation (NAO) is one of the most prominent large-scale patterns of atmospheric variability over the middle and high latitudes of the northern hemisphere. It exerts a dominant influence upon climate variability on various time scales, from weeks to decades, over the Atlantic sector and across wide regions of North America, the Arctic, Eurasia and the Mediterranean.

Interannual oscillatory modes in the North Atlantic region in both the ocean and the atmosphere have been identified in many observational studies (e.g., Bjerknes 1964; Deser and Blackmon 1993; Dettinger et al. 1995; Hurrell and Van Loon 1997; Moron et al. 1998; Da Costa and Colin de Verdière 2002; Feliks et al. 2010; Jajcay

\footnotetext{
* Corresponding author address: A. Groth, Department of Atmospheric and Oceanic Sciences, University of California at Los Angeles, 405 Hilgard Avenue, Los Angeles, CA 90095, USA

E-mail: andreasgroth@atmos.ucla.edu

†Permanent affiliation: Department of Mathematics, Israel Institute of Biological Research, Ness Ziona, Israel

${ }^{\ddagger}$ Additional affiliation: Geosciences Department and Laboratoire de Météorologie Dynamique (CNRS and IPSL), Ecole Normale Supérieure, Paris, France
}

et al. 2016). The extent to which these oscillations are due to purely oceanic (Dijkstra and Ghil 2005; Berloff et al. 2007, and references therein), purely atmospheric (Frankignoul and Hasselmann 1977; Frankignoul et al. 1997) or coupled (Vannitsem et al. 2015, and references therein) processes is still a matter of controversy. Whatever the origin of these oscillations, understanding the complex interactions between the ocean and the overlying atmosphere is crucial for the prediction of low-frequency climate variability (Kushnir et al. 2002; Czaja et al. 2003; Hurrell et al. 2003; Visbeck et al. 2003; Liu 2012; Vannitsem et al. 2015; Pierini et al. 2016).

In this paper, we use the Simple Ocean Data Assimilation (SODA) reanalysis (Carton and Giese 2008; Giese and Ray 2011) to address the question of possible shared mechanisms of interannual variability between the North Atlantic Ocean and the atmosphere above and near it. In particular, we will address the following two questions:

(i) Can we extract statistically significant modes - either individual or joint - in both the atmosphere and the ocean by using advanced multivariate methods of spectral analysis? 
(ii) To what extent may such modes be associated with shared mechanisms of interannual variability?

Question (i) will be investigated in sections 3 and 4 of the paper, while question (ii) will be the object of sections 5 and 6.

To identify interannual variability in the extensive reanalysis data set, we rely here on multivariate singular spectrum analysis (M-SSA), an advanced method for the analysis of spatio-temporal datasets; please see Ghil et al. (2002) for a comprehensive review of M-SSA and Groth and Ghil $(2011,2015)$ for recent improvements thereof that help separate distinct spatio-temporal patterns. These improvements developed further herein study the ocean's response to variability in the atmospheric forcing.

A series of papers have studied the wind-driven doublegyre problem across a hierarchy of models, from simple rectangular-shaped ocean basins and 1.5-layer reduced gravity models (Jiang et al. 1995; Speich et al. 1995; Chang et al. 2001) to more realistic topographies and several layers Simonnet and Dijkstra (2002); Simonnet et al. (2003a,b); Dijkstra (2005); Dijkstra and Ghil (2005); Simonnet et al. (2005). These papers consistently found relaxation-oscillation-like response of the ocean on interannual time scales to time-independent wind forcing, either idealized or climatological.

In this paper, an objective test against red noise is therefore discussed to distinguish deterministic behavior from random fluctuations. The present analysis of oceanic and atmospheric datasets extends the information gained from this hierarchy of models insofar as the atmospheric forcing comes from an atmospheric reanalysis, called 20CR (Compo et al. 2011), with its own inherent spatiotemporal complexity, and the ocean models are replaced by the oceanic general circulation model that is used in the SODA reanalysis.

In the SODA reanalysis, the atmosphere is just a prescribed, time-and-space-dependent boundary condition for the ocean model. In this setting, variability in the ocean model cannot instantaneously feed back into the atmospheric model used for $20 \mathrm{CR}$, and that in turn prevents the atmosphere and the ocean from mutually adjusting their spatio-temporal behavior, as they would in a fully coupled ocean-atmosphere model. In this two-step approach, though, mutual adjustment is possible through assimilation of instrumental data.

Carton and Giese (2008) have, in fact, shown that the assimilation of ocean observations further improves the quality of the reanalysis, and that it reduces mismatches in eddy kinetic energy and sea level variability between independent observations and the ocean model's output in the presence of atmospheric forcing alone.

In the absence of ocean data assimilation, though, these authors have shown that such mismatches are more substantial. Despite a demonstrable improvement of the re- analysis that the assimilation of oceanic data produces, discrepancies between the temporal patterns in the atmospheric forcing and in the ocean dynamics may still persist. It is precisely these residual discrepancies that we aim to identify and understand in the present work by applying our improved M-SSA methodology.

To this end, we have devised a protocol related to the classical M-SSA null-hypothesis test (Allen and Smith 1996; Groth and Ghil 2015), to test the hypothesis of interannual variability in the ocean being a response to similar variability in the atmospheric forcing. The present method complements, therefore, the common approach of comparing multiple realizations of an ocean general circulation model, driven with different atmospheric forcings (e.g., Grégorio et al. 2015; Sérazin et al. 2015).

The remainder of the paper is organized as follows: In section 2, we present the SODA dataset and give a brief introduction to M-SSA. In section 3, we analyze the atmospheric and oceanic datasets in two separate M-SSA analyses and identify patterns of similar interannual variability. Then, in section 4 , we undertake a joint analysis of both datasets to obtain further insight into the spatiotemporal behavior of the coupled ocean-atmosphere dynamics. Discrepancies between the temporal patterns that we find in the atmospheric forcing and in the ocean's response are ascertained and analyzed in section 5. A summary of the results concludes the paper in section 6 , and four appendices provide technical details.

\section{Dataset and methods}

\section{a. The Simple Ocean Data Assimilation (SODA) dataset}

The SODA reanalysis of ocean climate variability used herein is version 2.2.4 of Giese and Ray (2011), and it extends over the 138-yr interval 1871-2008. Note that the original SODA dataset provided by Carton and Giese (2008) only extended over $50 \mathrm{yr}$ and it used forcing from the ERA-40 atmospheric reanalysis (Uppala et al. 2005).

In our study we focus on SODA's oceanic temperature field (TEMP), with a vertical resolution of 40 levels, and the sea surface height (SSH). The global ocean model used in SODA has an eddy-permitting horizontal resolution of $0.25^{\circ} \times 0.4^{\circ}$, and the output variables are then mapped onto a uniform grid with a resolution of $0.5^{\circ} \times 0.5^{\circ}$. The SODA reanalysis assimilates hydrographic and SST data into this model with surface boundary conditions from the atmospheric 20CRv2 reanalysis (Compo et al. 2011), from which we include the zonal surface wind stress (TAUX) in our analysis.

The monthly TEMP, SSH, and TAUX data fields are first converted into anomalies, i.e. we remove at each grid point the average value over the entire 138-yr interval. Since we are interested in variability on interannual time scales, we further eliminate variability at frequencies 
$f \geq 0.5$ cycles per year with a Chebyshev type-I low-pass filter from which we then take all $N=138$ July values.

Feliks et al. (2013) showed in their appendix that such a Chebyshev filter comes very close to an ideal low-pass filter, with practically no distortions of either phase or amplitude in the passband, i.e. below the Nyquist frequency of $f_{\mathrm{N}}=0.5$ cycles per year, and a highly effective damping in the stopband of $f \geq 0.5$ cycles per year. The more customary 12-month or 3-month averages, on the other hand, are strongly frequency dependent and therefore influence any subsequent spectral analysis; see the discussion of Figs. A1 and A2 in Feliks et al. (2013) for additional details.

Figure 1 displays the spatial patterns of the mean field (contour lines) and of the standard deviation of interannual variability (color) in the North Atlantic region of interest, taken here to be $\left(25^{\circ} \mathrm{N}-65^{\circ} \mathrm{N}, 80^{\circ} \mathrm{W}-0^{\circ}\right)$. The Gulf Stream front is very prominent in the two ocean variables of SSH and SST variability (panels $b$ and c); it appears as a strong gradient in the mean field and also as a narrow, meandering ridge of interannual variability.

The front is aligned with the Florida Current, which flows along the east coast of North America until the separation point at Cape Hatteras; it then extends into the open ocean to $40^{\circ} \mathrm{W}$, beyond which longitude it weakens and the mean-field contour lines diverge. This divergence is associated with the birth of the Azores Current that flows toward the southeast and the North Atlantic Drift flowing northeastward.

The contour lines of the TAUX mean-field (panel a), on the other hand, show a typical pattern of mid-latitude westerlies and lower-latitude easterlies. The pattern of interannual variability on top of it (color) reaches its maximum at about $60^{\circ} \mathrm{N}$, and contributes to a strengthening or weakening of the westerlies.

We have also analyzed the meridional component of the 20CRv2 surface wind stress (TAUY), but found its interannual variability (not shown) to possess much smaller variance over the North Atlantic basin. To simplify the MSSA analysis, we therefore focus in this paper on TAUX, and only discuss TAUY whenever necessary.

\section{b. Multivariate singular spectrum analysis (M-SSA)}

To identify complex patterns of spatio-temporal behavior in the extensive SODA reanalysis datasets, we rely here on M-SSA, which provides an efficient and robust tool to extract dynamics from short, noisy time series. M-SSA relies on the classical Karhunen-Loève decomposition of stochastic processes into data-adaptive orthogonal functions. Broomhead and King (1986b) introduced M-SSA into dynamical system analysis as a robust version of the Mañé-Takens idea to reconstruct the underlying dynamics from a time-delayed embedding of time series; see Ghil et al. (2002) and Alessio (2016, chapter 12) for a comprehensive overview of the methodology and of related spectral methods. Appendix A here presents some of the requisite mathematical details.

The M-SSA method essentially diagonalizes the lagcovariance matrix to yield a set of orthogonal eigenvectors and the corresponding eigenvalues. The eigenvectors are also referred to as space-time empirical orthogonal functions (ST-EOFs), while the eigenvalues equal the variance that is captured in the direction of a given ST-EOF. Oscillatory behavior is reflected in so-called oscillatory pairs, i.e. ST-EOFs with approximately equal eigenvalues and fundamental frequencies (Vautard and Ghil 1989; Plaut and Vautard 1994). To improve the separability of distinct frequencies, we rely here on a subsequent varimax rotation of the ST-EOFs (Groth and Ghil 2011).

The presence of such oscillatory pairs, though, is not sufficient in order to reliably identify deterministic behavior in the underlying dynamics. Allen and Smith (1996) provided, therefore, an objective Monte Carlo-type hypothesis test against red noise for the statistical significance of eigenvalues in the single-channel case, and Allen and Robertson (1996) suggested a multichannel extension thereof to M-SSA.

The latter authors, however, already stated that this test may become too lenient and less specific as a consequence of the variance-compression problem. In this context, Groth and Ghil (2015) found the Procrustes algorithm helpful in comparing the data eigendecomposition with that of the surrogate data generated by the red-noise null hypothesis, and showed that application of this algorithm substantially improves the explanatory power of the test. These authors further provided a rigorous mathematical extension of a composite null hypothesis test for M-SSA: for instance, a strong trend component can be excluded from the raw data when estimating the parameter of the null hypothesis.

In the analysis of extensive datasets, such as the SODA dataset at hand, it is common practice to first project the dataset onto a subset of spatial EOFs (S-EOFs) by means of a conventional principal component (PC) analysis. This compression of the dataset into a few leading PCs is meant to reduce the computational costs of a subsequent M-SSA analysis (e.g., Dettinger et al. 1995; Allen and Robertson 1996; Robertson 1996); see also Moron et al. (1998) and Table 1 therein.

Groth and Ghil (2015), though, have demonstrated possible negative effects of this variance compression on the detection rate of weak signals when the number of retained PCs becomes too small. In appendix B, we demonstrate that this practice can further affect negatively the judicious reconstruction of the spatio-temporal patterns of such weak signals, and recommend retaining a maximum number of PCs. Moreover, we show that the M-SSA anal- 
(a) TAUX $\mid \max =0.038 \mathrm{~N} \mathrm{~m}^{-2}$

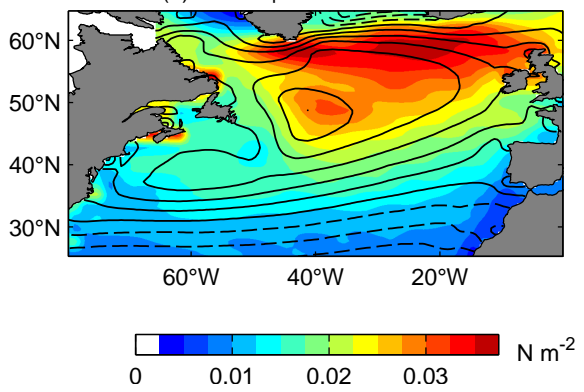

(b) $\mathrm{SSH} \mid \max =0.37 \mathrm{~m}$

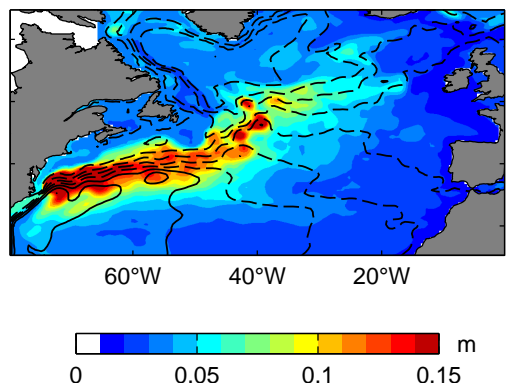

(c) SST $\mid \max =2.2^{\circ} \mathrm{C}$

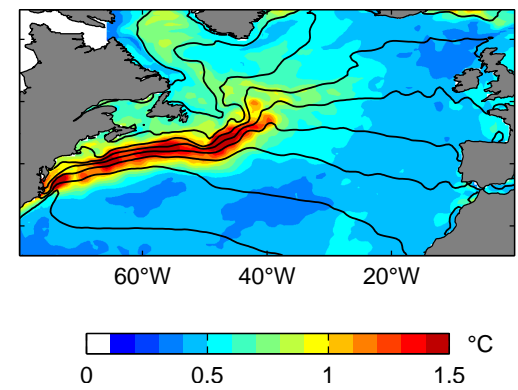

FIG. 1. Climatological properties of (a) TAUX, (b) SSH, and (c) SST anomalies in the North Atlantic basin $\left(25^{\circ} \mathrm{N}-65^{\circ} \mathrm{N}, 80^{\circ} \mathrm{W}-0^{\circ}\right)$; the SST is taken to equal the temperature in the upper $5 \mathrm{~m}$ of the SODA TEMP dataset. Colors correspond to interannual variability (standard deviation), with extreme values given in the legend (max). Contour lines correspond to the TAUX, SSH, and SST mean fields, with a contour interval (CI) of $0.015 \mathrm{~N} \mathrm{~m}^{-2}, 0.14 \mathrm{~m}$, and $2.5^{\circ} \mathrm{C}$, respectively. Solid and dashed lines correspond to positive and negative anomaly values in panels (a,c), and to differences from the mean sea level in (b).

ysis on PCs is mathematically equivalent to that on the full gridded dataset, when the full number of PCs is retained.

To combine different physical quantities in a single $\mathrm{M}$ SSA analysis, we normalize the annually sampled TAUX, SSH, and TEMP anomalies to unit variance, i.e. we divide each dataset by its corresponding standard deviation. Furthermore, to account for geographical variations in the grid-size resolution, we multiply the anomalies at each grid point by the square-root of the cosine of its latitude. Throughout the whole paper, physical units are restored by an inverse transformation when presenting patterns of interannual variability.

\section{Individual oscillatory modes}

First, we study the atmospheric and oceanic datasets in two separate M-SSA analyses. Doing so will give us a first look at whether there are significant similarities among the interannual variabilities of the TAUX, SSH, and TEMP anomalies, but also highlight possible differences.

\section{a. Wind-stress modes}

We start with an M-SSA analysis of the TAUX anomalies. The annually sampled dataset of length $N=138$ is first projected onto the full set of S-EOFs, which gives a total of $L=138$ PCs as input channels for the analysis.

The M-SSA results are shown in Fig. 2, with the spectrum of eigenvalues and the corresponding significance levels in panel (a). The latter levels have been derived from a composite null hypothesis of the leading ST-EOF 1 plus AR(1) noise; see Groth and Ghil (2015) for more methodological details.

In the significance test, we have chosen to exclude STEOF 1 as it clearly exceeds the other ST-EOFs in its variance. The corresponding reconstruction of ST-EOF 1, which is projected onto its leading S-EOF for illustration, shows a strong trend that is clearly visible early in the dataset, cf. panel (b). To which extent this trend could be attributed to changes in the availability of data in the atmospheric reanalysis or to natural variability, though, remains an open question.

The remainder of the ST-EOFs is then tested against AR(1) noise, in which two oscillatory pairs clearly emerge as significant in our Monte Carlo test at the 99\% level; their frequencies are indicated by the dashed lines in panel (a). These two pairs represent quasi-biennial variability, with a period of $2.7 \mathrm{yr}$ and $2.2 \mathrm{yr}$, respectively. The reconstruction of the 2.7-yr mode (panel c) shows that this mode is most active in the second half of the dataset, $\gtrsim 1940$, and that it almost vanishes in the first half, $\lesssim 1940$. The reconstruction of the 2.2-yr mode (panel e), on the other hand, shows activity throughout the whole time interval, although it it is slightly modulated and reaches its maximum near 1950.

It appears that no further ST-EOFs exceed the $99 \%$ significance threshold. There is, however, one oscillatory pair with a period of $7.7 \mathrm{yr}$ that appears close to the upper significance level, and that is significant at the $95 \%$ level. This alone is not sufficient to pay it greater attention, but we do have prior reason to focus on atmospheric and oceanic variability in the 7-8-yr frequency band for the North Atlantic (e.g., Moron et al. 1998; Da Costa and Colin de Verdière 2002; Gámiz-Fortis et al. 2002; Paluš and Novotná 2004; Feliks et al. 2013); hence we reran the analysis so that it enhances the signal-to-noise ratio in this particular case.

The reconstruction of the 7.7-yr mode in panel (d) shows a decline in variability as time advances past the mid-20th century, and we therefore restrict the analysis in a second run to the time interval 1871-1950. This modification increases the relative variance that this mode captures and the null hypothesis can be indeed rejected at a higher level of 98\% (not shown). 
(a) TAUX | Eigenvalues

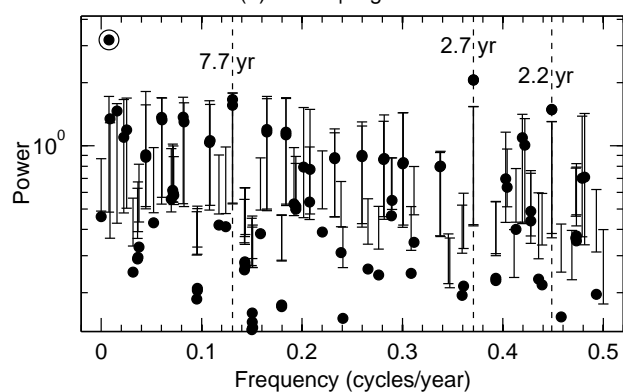

(b) $\mathrm{RC} 1 \mid 4.9 \%$

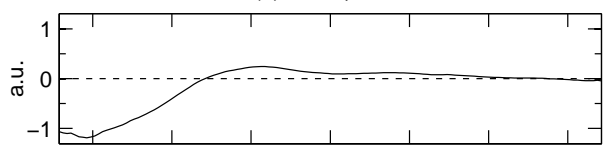

(c) RCs $2-3|2.7 \mathrm{yr}| 6.3 \%$

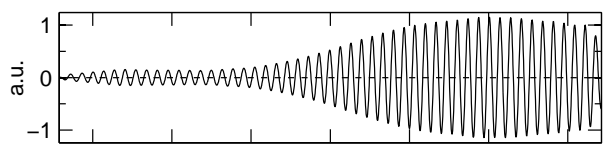

(d) RCs 4-5 | $7.7 \mathrm{yr} \mid 4.9 \%$

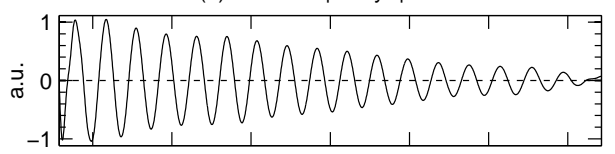

(e) RCs $6-7|2.2 \mathrm{yr}| 4.6 \%$

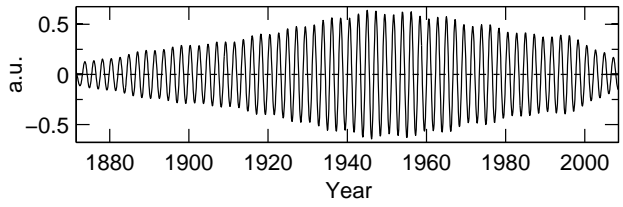

FIG. 2. Spectral properties of the North Atlantic TAUX anomalies. The M-SSA analysis here uses a window length of $M=40 \mathrm{yr}$; the subsequent varimax rotation uses ST-EOFs $2-40$, while ST-EOF 1 is excluded. (a) The estimated variance in each mode is shown as black dots, plotted as a function of their corresponding frequency. Lower and upper ticks on the error bars correspond to the $1 \%$ and $99 \%$ quantiles from a Monte Carlo test that uses the scaled target-rotation algorithm of ST-EOFs; the test ensemble has 1000 members. In the composite null hypothesis, the leading ST-EOF (target dot) is excluded from the test and the remaining ST-EOFs are tested against AR(1) noise. (b-e) Reconstruction of the TAUX field with ST-EOF 1, and three oscillatory pairs at a period length of $2.7 \mathrm{yr}, 7.7 \mathrm{yr}$, and $2.2 \mathrm{yr}$, respectively; each mode is projected onto its leading S-EOF. The variance captured by each mode is given in the legend of each panel (in percent).

In summary, we are thus able to identify three oscillatory modes in the TAUX anomalies, with a period of $7.7 \mathrm{yr}, 2.7 \mathrm{yr}$, and $2.2 \mathrm{yr}$, respectively. These findings are consistent with numerous other publications that have presented interannual variability in the atmospheric fields over the North Atlantic basin (see e.g. Rogers 1984; Hurrell 1995; Hurrell and Van Loon 1997; Robertson 2001; Feliks et al. 2010). In particular, these findings are consistent with the global presence of the $7-8$-yr mode in the in- terannual climate variability for the North Atlantic, North America, Europe, and Eastern Africa (see e.g. Kondrashov et al. 2005; Hanrahan et al. 2009; Feliks et al. 2013; Jajcay et al. 2016).

\section{b. Oceanic modes}

To which extent is the interannual variability in the TAUX anomalies also reflected in ocean dynamics? To find out, we analyze the TEMP and SSH anomalies in a joint M-SSA analysis and first combine both annually sampled datasets into a multivariate time series with a much larger number of channels. Note that each dataset has been normalized and latitude-weighted as explained in section 2, prior to a projection onto the full set of SEOFs, yielding finally $L=138 \mathrm{PCs}$ as input channels for the M-SSA analysis.

The combined M-SSA results for the TEMP and SSH anomalies are plotted in Fig. 3. The eigenvalue spectrum and the corresponding significance levels are given in panel (a); these levels are based on a composite null hypothesis of low-frequency variability in ST-EOFs $1-4$ plus $\mathrm{AR}(1)$ noise.

The reconstruction with ST-EOFs 1-4 (panel b) shows low-frequency behavior with a minimum at around 1910 and an irregular increase thereafter. In the group of STEOFs 1-4, we observe a pairing in ST-EOFs 2-3, which could be linked to multidecadal behavior with a $60-70-y r$ period. The chosen window length of $M=40 \mathrm{yrs}$, though, together with the presence of two cycles only, limits the reliability of detecting such long-term behavior.

Still, it is interesting to remark that this mode contributes to a decline in the trend at the end of the time interval, roughly after 2005. Such low-frequency variability in turn can limit our ability to predict the persistence of the secular warming trend, as stated already by Ghil and Vautard (1991).

In Fig. 3a, we identify several modes that emerge above their upper significance level. Among them are three modes which agree rather well in period with the three modes found to be significant in TAUX, namely $7.7 \mathrm{yr}$, $2.6 \mathrm{yr}$, and $2.3 \mathrm{yr}$, although the ordering by variance of the first two differs. The reconstructions of these three oscillatory modes in panels $(\mathrm{c}-\mathrm{e})$ resemble to some extent those of the TAUX anomalies, although interesting differences are visible. We will return to these differences in greater detail in section 5 .

The present analysis yields, furthermore, ST-EOFs that are significant in the combined TEMP-SSH anomalies, but which lack an equivalent significant mode in TAUX. A 13-yr oscillation, for example, agrees well in periodicity and geographical extent (not shown), with decadal variability found in the North Atlantic SST field; see Moron et al. (1998) for an overview. Deser and Blackmon (1993) found a similar pattern of decadal variability in the SST 

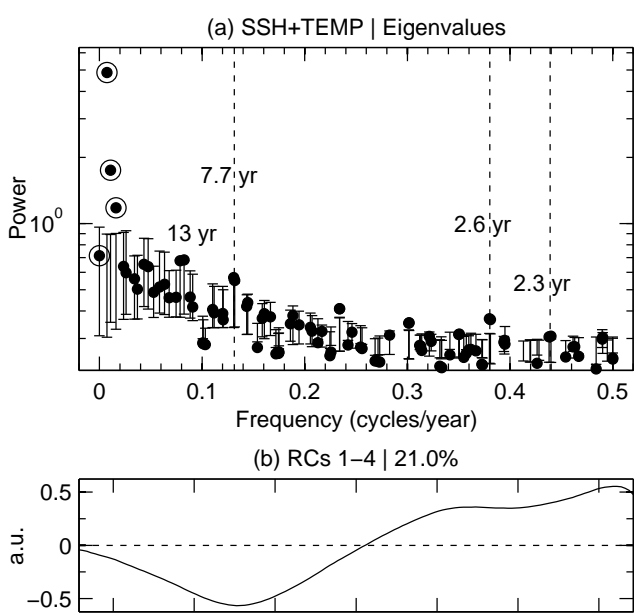

(c) RCs 33-34 | $2.6 \mathrm{yr}$ | 1.8\%

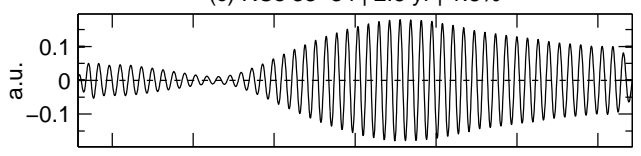

(d) RCs $11-12$ | $7.7 \mathrm{yr} \mid 2.7 \%$

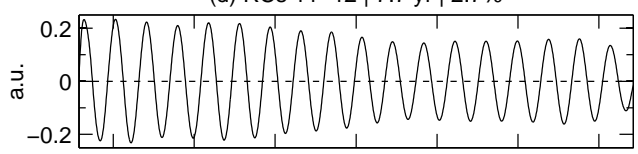

(e) RCs $48-49$ | $2.3 \mathrm{yr} \mid 1.5 \%$

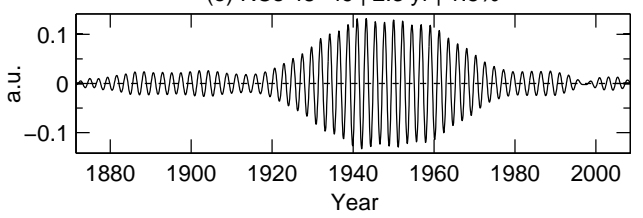

FIG. 3. Same as Fig. 2, but for TEMP and SSH anomalies from a joint M-SSA analysis; the subsequent varimax rotation uses ST-EOFs 3-50. In the composite null hypothesis, the leading ST-EOFs 1-4 (target dots) are excluded from the test and the remaining ST-EOFs are tested against $\mathrm{AR}(1)$ noise.

and TAUX fields; this pattern, though, was not statistically significant in their study.

The number of significant interannual-variability modes in the SODA dataset provides additional food for thought in dealing with the question - already mentioned at this paper's beginning - of whether these modes arise from intrinsic ocean dynamics alone (Jiang et al. 1995; Dijkstra and Ghil 2005; Berloff et al. 2007; Simonnet et al. 2005; Pierini et al. 2016); through the ocean's linear (Frankignoul and Hasselmann 1977; Frankignoul et al. 1997) or nonlinear (Dewar 2003) response to atmospheric fluctuations; or from genuinely coupled modes (Vannitsem et al. 2015, and references therein).

In the present M-SSA analysis, we focus on the conceptually simplest hypothesis of a linear relationship, and continue to examine the three modes that we found to have similar periods in both media: the 7-8-yr mode and the two quasi-biennial modes, cf. Figs. 2 and 3. The restriction to linearity is for the sake of simplicity, and not because of an essential limitation of the methodology to the analysis of linear systems alone. Thus, for instance, Groth and Ghil (2011) found M-SSA helpful in the synchronization analysis of coupled chaotic oscillators.

\section{Joint ocean-atmospheric modes}

Next, we proceed with a joint M-SSA analysis of the TAUX, SSH, and TEMP anomalies to better understand the spatio-temporal structure associated with interannual variability in the wind stress and the North Atlantic temperature field. As before, we combine the three annually sampled and normalized datasets into a single multivariate time series; projection onto its full set of S-EOFs yields the $L=138$ PCs as input channels for our M-SSA analysis, cf. appendix B.

In this sequential approach - starting with separate MSSA analyses of TAUX and TEMP-SSH anomalies, and then combining all anomalies into a single M-SSA analysis - the limitations of a decomposition into spatially orthogonal functions become clearly visible. Just as the number of EOFs is limited by the rank of $\mathbf{x}$, so is the capacity of M-SSA to separate between different spectral components. This limitation emerges in particular when the number $D$ of channels is much larger than the length $N$ of the time series.

It is therefore not surprising that - in the M-SSA analysis on the combined fields, atmospheric and oceanic we found again three significant oscillatory modes, which exhibit the periods of $7.7 \mathrm{yr}, 2.7 \mathrm{yr}$, and $2.2 \mathrm{yr}$ that were identified in the two separate M-SSA analyses above. This result shows that differences between the ST-EOFs of the TAUX anomalies and those of the TEMP-SSH anomalies are sufficiently small for their joint M-SSA analysis to indicate joint interannual variability in all three fields.

We will return to this limitation of M-SSA, and to a more detailed analysis of the differences in the oscillatory behavior of the atmospheric and oceanic fields, in section 5. The joint M-SSA analysis, though, is mathematically equivalent to a total-least-square regression of the three fields onto common oscillatory modes, in which the joint RCs (not shown) resemble an average of the separate RCs in Figs. 2 and 3.

\section{a. Spatial structure}

The spatial structure of interannual variability in the three joint modes with periods of $7.7 \mathrm{yr}, 2.7 \mathrm{yr}$, and 2.2 yr is shown in Fig. 4. The variability is plotted in physical units that correspond to the standard deviation of the reconstructed components (RCs) resulting from the M-SSA.

In the standard deviations of the TAUX anomalies (left column), we observe in all three modes a fairly homogeneous pattern, with a single large maximum extending 
(a) TAUX | 7.7yr $\mid \max =0.011$

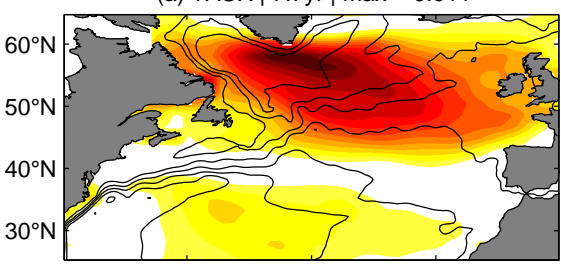

(d) TAUX $|2.7 \mathrm{yr}| \max =0.013$

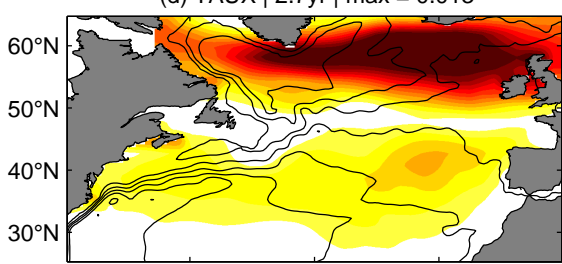

(g) TAUX | 2.2yr $\mid \max =0.0076$

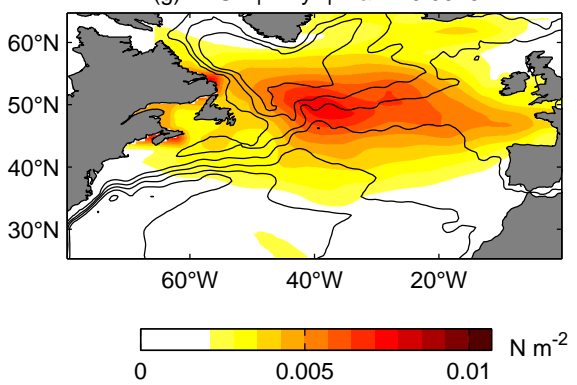

(b) SSH $|7.7 y r| \max =0.068$

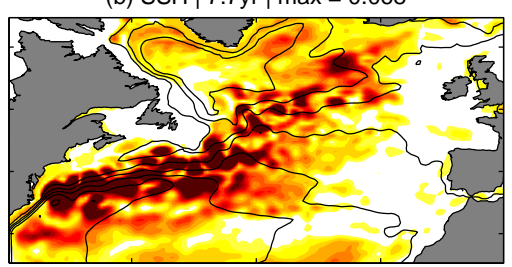

(e) SSH $|2.7 y r| \max =0.04$

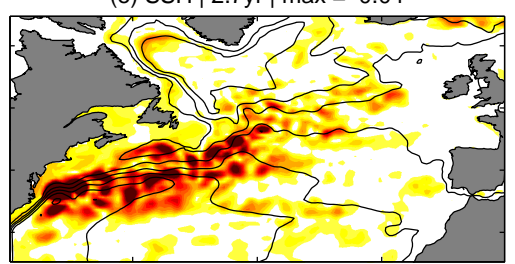

(h) SSH $|2.2 \mathrm{yr}| \max =0.032$

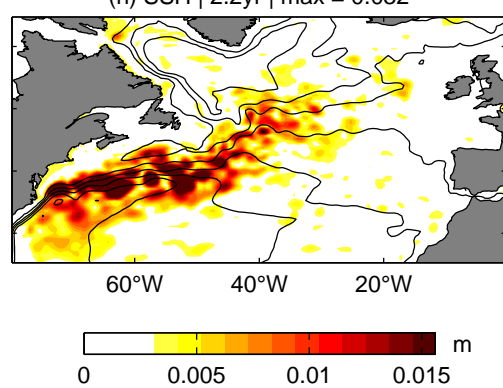

(c) SST | 7.7yr | $\max =0.44$

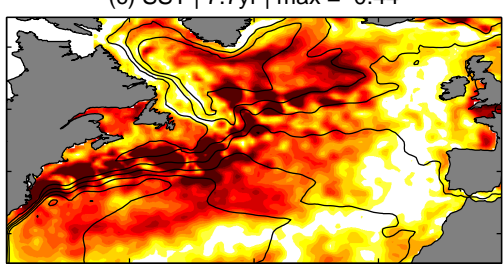

(f) SST | 2.7yr $\mid \max =0.41$

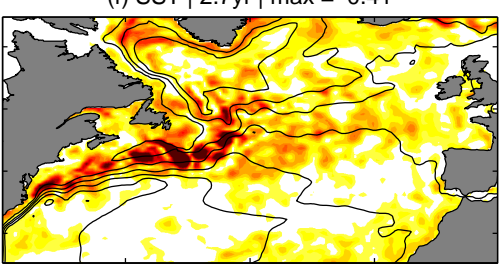

(i) SST $|2.2 \mathrm{yr}| \max =0.28$

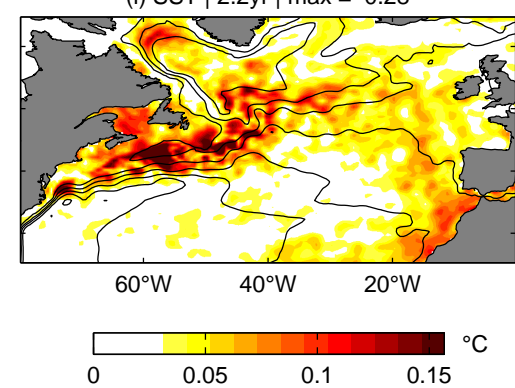

FIG. 4. Spatial structure of interannual variability in the three joint oscillatory modes with periods of (a-c) 7.7-yr, (d-f) 2.7-yr, and (g-i) 2.2-yr. Shown is the standard deviation of the corresponding reconstructed components (RCs) resulting from an M-SSA analysis with a window length of $M=40 \mathrm{yr}$ and a subsequent varimax rotation of ST-EOFs 3-50; see appendices A and B for a review of the methodology. The standard deviations in the anomalies of (a, d, g) TAUX, (b, e, h) SSH, and (c, f, i) SST are plotted in color, and they are transformed back into physical units. Extreme values are given in the legend (max). The contour lines in all nine panels correspond to the SSH mean field, with a contour interval (CI) of $0.2 \mathrm{~m}$.

over much of the subpolar gyre. This pattern is most intense and lies farthest north in the 2.7-yr mode, and it is weakest and lies farthest south, astride the North Atlantic Drift, in the 2.2-yr mode. In all three modes, this pattern is located in fairly high latitudes, i.e. in a region in which interannual variability in TAUX is generally high, cf. Fig. 1a.

In the standard deviations of the SSH and SST anomalies, plotted in the middle and right column of Fig. 4, respectively, we observe much smaller-scale features lying mainly along the Gulf Stream front. The scale of these features seems to correspond to the meander-andeddy pattern associated with the Gulf Stream's instabilities, as shown also by Figs. 1b,c.

For the 2.7-yr and 2.2-yr modes, the intense variability is limited by-and-large to the Gulf Stream region and extends only little further, along the North Atlantic Drift, cf. panels (e,f) and (h,i). But in the 7.7-yr mode - in the SSHs and especially in the SSTs, Figs. 4b,c, respectively - we observe, beside the intense variability along the Gulf Stream front, a larger-scale pattern of high variability that covers large areas south and east of the front.
This spatio-temporal pattern starts in the Florida Current and continues further east and north of Cape Hatteras, especially in the SST anomalies (panel c), along the North Atlantic Drift, to finally cover most of the subpolar gyre. Although the amplitude of the SST standard deviation in this mode only reaches about $0.15{ }^{\circ} \mathrm{C}$ in absolute value, it captures up to $13 \%$ of the total interannual variability in this active region; when excluding the low-frequency behavior in ST-EOFs 1-4, the variance captured is as high as $25 \%$. This finding shows that, especially off the Gulf Stream, the fraction of variance associated with the 7.7yr mode can be much larger than the roughly $2.7 \%$ that this mode captures on average in the entire North Atlantic TEMP field, cf. Fig. 3d.

\section{b. Dynamical behavior}

To understand the dynamical properties that underlie the spatial structure seen in Fig. 4, we construct next the so-called phase composites of the oscillatory modes (Moron et al. 1998). Thats is, the successive maps associated 
with a given mode's RCs are divided into eight phase categories and one averages in time over all the maps in each category to obtain the phase composite of that category; see appendix $\mathrm{C}$ for the specific procedure used herein.

Figure 5 shows the corresponding eight phases for the 7.7-yr mode in the SST anomalies. Along the Gulf Stream front, we observe localized coherent structures of large amplitude, up to roughly $\pm 0.6^{\circ} \mathrm{C}$. The small characteristic scale and the large amplitude of this pattern is in agreement with the generally high variability in this region. In addition, we observe the SW-NE propagation of a wave off the front. The propagating wave starts near the Florida Current, attains $0.2^{\circ} \mathrm{C}$ in absolute value, covers large areas south and east of the front, and reaches the subpolar gyre after a full cycle.

Similar oscillatory patterns have already been reported in the analysis of other instrumental SST records for the North Atlantic basin. Sutton and Allen (1997), for example, have identified in their analysis of shipboard observations a northward propagation of SST anomalies with a propagation speed similar to that of our analysis. Their dominant spectral peak, though, was centered at 12-14 yr in the SST power spectrum, i.e. similar to that of Deser and Blackmon (1993).

Moron et al. (1998) carried out an M-SSA analysis of North Atlantic SST anomalies from the UK Meteorological Office and were able to identify two separate standingwave modes, with periods of $13 \mathrm{yr}$ and $7.5 \mathrm{yr}$, respectively. In our analysis, though, only the 13 -yr mode shows a standing wave with a similar geographical extent as in Moron et al. (1998) (not shown). The apparent discrepancy between the 7.7-yr traveling wave found here and the standing-wave character of the 7.5-yr mode of Moron et al. (1998) may be due to their strong compression of the UK dataset onto a few leading S-EOFs prior to M-SSA analysis. This possibility is discussed in appendix B here, in connection with Fig. B1.

Still, the region occupied by the 7.5-yr mode of Moron et al. (1998) agrees well with the region in which our 7.7yr mode is active. Moreover, in an analysis of the same UK dataset, Simonnet et al. (2005) identified a northwardpropagating mode with a period of 7-8 yr, while Da Costa and Colin de Verdière (2002) and Jamison and Kravtsov (2010) found a similar mode in the dataset of Kaplan et al. (1998).

Figure 6 shows the corresponding eight phases of the 7.7-yr mode in the TAUX anomalies (in color). The oscillatory pattern for the zonal wind stress is essentially characterized by a meridional-dipole structure, with a much stronger northern pole. The eight phases reflect a standing wave, with in-phase behavior over large regions, and the dipole's up-and-down seasaw leads to an alternative weakening and strengthening of the westerlies north of $40^{\circ} \mathrm{N}$.

Phase composites of the vector wind stress (arrows) have been reconstructed by a projection of the TAUY anomalies onto the joint M-SSA analysis of TAUX, SSH, and TEMP anomalies; see appendix D for the details of the algorithm. This analysis clearly illustrates the great intensity of coherent TAUX variations over the subpolar gyre, but it also shows that the wind pattern in our 7.7-yr mode resembles to some extent the atmospheric dipole pattern identified by Deser and Blackmon (1993). In agreement with their analysis, a comparison of the phase composites in the SST and TAUX anomalies of Figs. 5 and 6, respectively, shows that, at higher latitudes, positive TAUX anomalies correspond to negative SST anomalies; see also the supplementary animation. Sutton and Allen (1997) have also identified similar patterns of sea surface cooling by the wind stress in higher latitudes.

This cooling effect is physically quite plausible but ascertaining its exact mechanism will require a study of the ocean-atmosphere heat fluxes - sensible and latent that goes beyond the scope of the current paper. In the meantime, it is interesting to note that the corresponding phase composites in the SSH anomalies (not shown) resemble in many of their details the SST anomalies; i.e. we observe covariations between SST and SSH in this 7-8-yr traveling wave.

To illustrate the dynamical structure of an oscillatory mode in a single picture, we continue next with the calculation of phase maps, as described in greater detail in appendix C. For each of the three oscillatory modes that we found, it is possible to identify in a unique way a spatial phase $\theta_{d}$ that puts - for each cycle of the oscillation, each variable (TAUX, SSH and SST) and each channel $d$ - the standard interval $[0,2 \pi]$ in one-to-one correspondence with the period of the oscillation $[0, T]$. As done for the phase composites, and for each oscillation, the origin of the phase $\theta_{d}$ is chosen arbitrarily, and phase maps are drawn with respect to this arbitrary origin $\theta_{d}^{(0)}$ and for one of the variables, TAUX, SSH or SST, which is kept fixed.

The resulting phase maps are given in Figs. 7(a-i) in the same $3 \times 3$ format as in Fig. 4 , for the TAUX, SSH, and SST anomalies and for each of the three oscillatory modes with periods of $7.7 \mathrm{yr}, 2.7 \mathrm{yr}$, and $2.2 \mathrm{yr}$. In the 7.7-yr mode (top panels), the spatial structure of the phase maps matches that of the already discussed phase composites in Figs. 5 and 6. That is, in the TAUX anomalies (panel a), the strong in-phase behavior over large homogeneous regions appears as large patches of similar colors, with phase opposition across the $40^{\circ} \mathrm{N}$ parallel, thus highlighting the meridional-dipole structure of the wind stress pattern in this mode. The SW-NE-oriented propagation in the SST anomalies (panel c), is reflected in a more gradual shift that runs through all the colors, with a zero isochron that runs WNW-ESE, roughly from New Foundland to the Canaries. The corresponding phase map in the SSH anomalies (panel b) appears remarkably close to that of the SST anomalies (panel c), and thus indicates substantial covariation between the SSH and SST fields. 


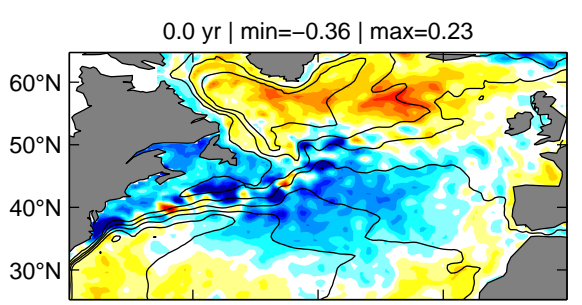

$2.9 \mathrm{yr}|\min =-0.29| \max =0.48$

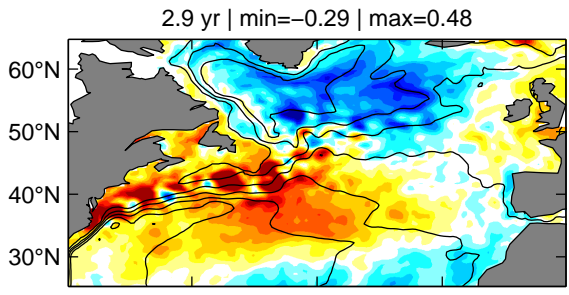

$5.8 \mathrm{yr}|\min =-0.57| \max =0.4$

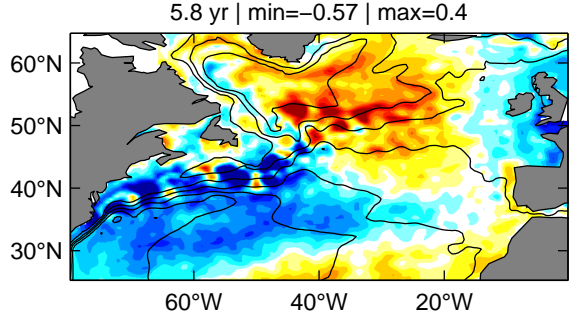

$1.0 \mathrm{yr}|\min =-0.43| \max =0.35$

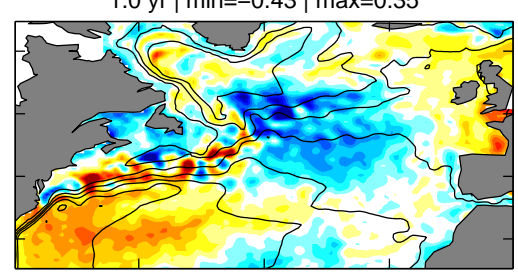

$3.8 \mathrm{yr}|\min =-0.22| \max =0.35$

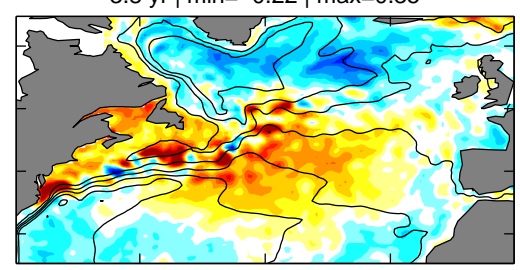

$6.7 \mathrm{yr}|\min =-0.51| \max =0.32$

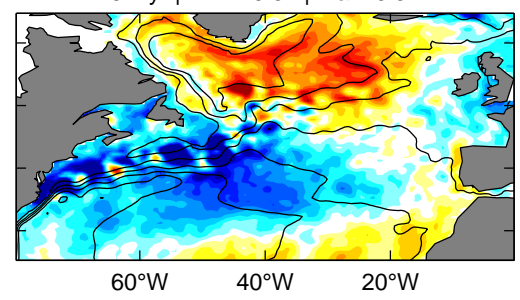

$1.9 \mathrm{yr}|\min =-0.39| \max =0.55$

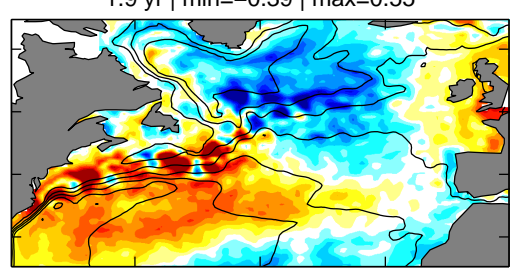

$4.8 \mathrm{yr}|\min =-0.35| \max =0.44$

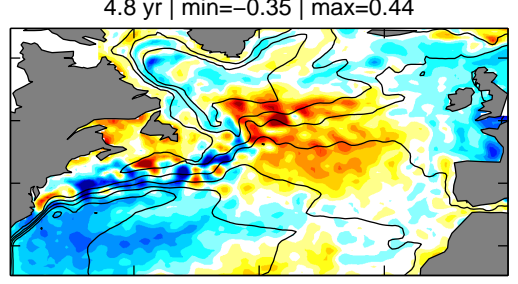

FIG. 5. Phase composites of the SST anomalies for the 7.7-yr oscillatory mode from a joint M-SSA analysis of TAUX, SSH, and TEMP anomalies, cf. Fig. 4. The eight phase categories are labeled by their midpoint (in years) in the legend of each panel and are shown in color; also listed in the legends are the minimum and maximum (min and max) amplitude of the anomalies, in degrees $\mathrm{K}$. The contours in all nine panels correspond to the SSH mean field, with $\mathrm{CI}=0.2 \mathrm{~m}$, as in Fig. 4.

In the 2.7-yr mode, we find a standing wave in TAUX (panel d), with a meridional dipole structure similar to the one seen in the 7.7-yr mode (panel a). The 2.7-yr mode, however, is displaced noticeably further north, with phase opposition across a parallel that is closer to $50^{\circ} \mathrm{N}$. This mode, moreover, displays no phase opposition between TAUX (panel d) and SST (panel f), nor does there appear to be noticeable covariation between the latter and SSH (panel e). Indeed, the phase dynamics in this mode's SST and SSH fields appears to be characterized by a more complex spatial structure.

In the 2.2-yr mode, we observe a north-to-south propagation in the TAUX anomalies (panel g), with no dipole structure like the one found in the two other modes. To some extent, the corresponding phase map in the SST anomalies (panel i) can be seen to be in phase opposition over much of the northern part of the domain, although not as obviously so as in the 7.7-yr mode. A comparison of the SSH and SST phase maps in panels (h) and (i), on the other hand, gives no indication of covariation.

Note that in both modes of quasi-biennial variability, the zonal component dominates the wind stress, as it does in the 7.7-yr mode in Fig. 6, while the meridional component plays only a minor role.
In the remainder of the paper, we try to gain a better understanding of the differences among the dynamical properties of the three oscillatory modes that could have caused the differences we noted in Fig. 7. In the next subsection, we examine the full TEMP field and discuss the effect of covariation across ocean layers. The whole of section 5 is then devoted to the questions of whether the oscillatory modes in the SST can be attributed entirely to variations in the wind stress forcing.

\section{c. Vertical phase coherence}

There are several mechanisms that could contribute to some degree of covariation between the SSH and SST fields; see for example Jones et al. (1998) and references therein. There is, for example, the possibility of covariation in specific geographic regions associated with strong mesoscale variability.

Lee and Cornillon (1995) had found, for instance, a dominant period of 9 months in the SST field measured by satellite infrared imagery along the Gulf Stream front. Such intraseasonal periodicities were then studied theoretically by Feliks and Ghil (1996, 1997), among others. Using an M-SSA analysis of SST observations and modeloutput data, Schmeits and Dijkstra (2000) identified co- 
$0.0 \mathrm{yr}|\min =-0.0035| \max =0.0067$

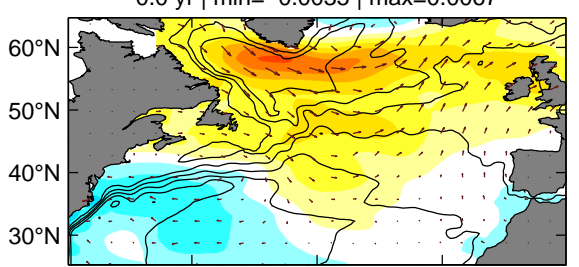

$2.9 \mathrm{yr}|\min =-0.0035| \max =0.0053$

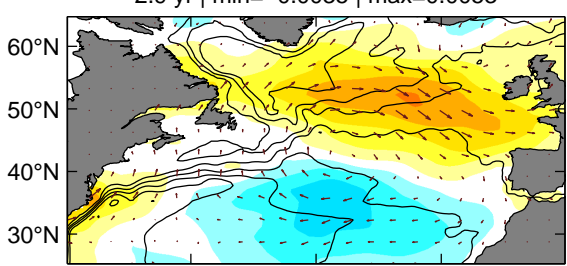

$5.8 \mathrm{yr}|\mathrm{min}=-0.011| \max =0.0046$

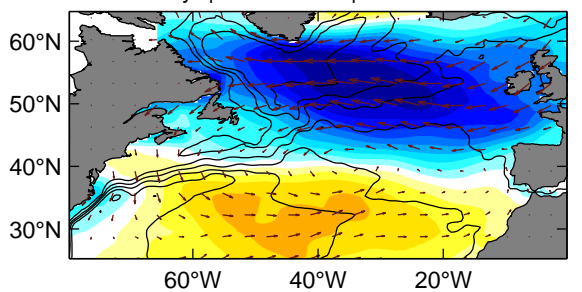

$1.0 \mathrm{yr}|\min =-0.0044| \max =0.012$

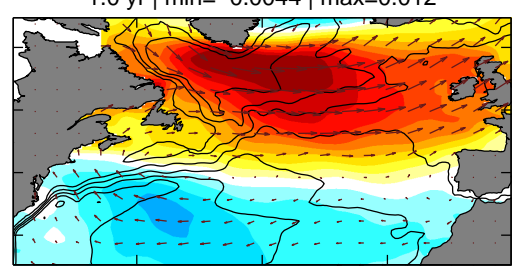

$3.8 \mathrm{yr}|\min =-0.0078| \max =0.0031$

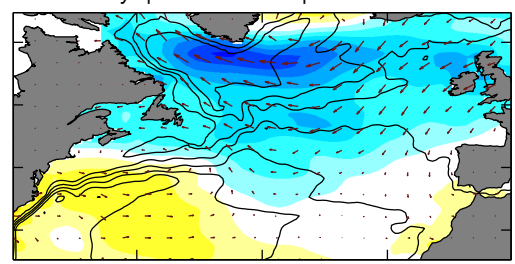

$6.7 \mathrm{yr}|\min =-0.006| \max =0.0038$

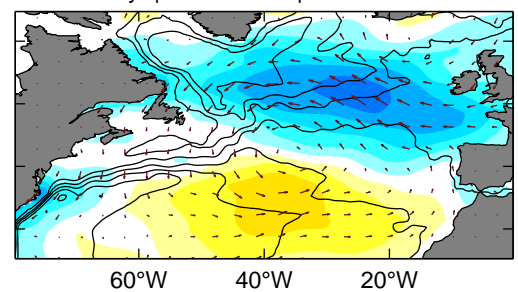

$1.9 \mathrm{yr}|\mathrm{min}=-0.0045| \max =0.011$

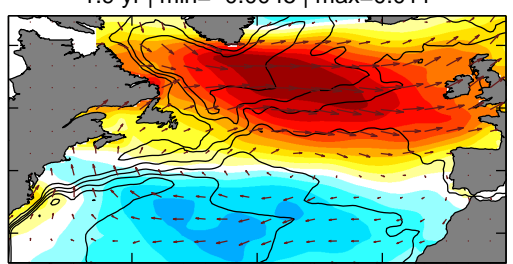

$4.8 \mathrm{yr}|\min =-0.013| \max =0.0046$
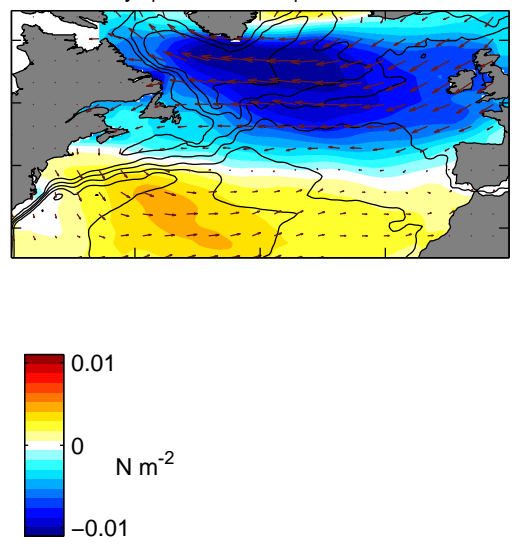

FIG. 6. Same as Fig. 5, but for TAUX anomalies (in color). The superimposed phase composites of the vector wind stress (arrows) are reconstructed using a projection of the TAUY anomalies onto the joint M-SSA analysis of the TAUX, SSH, and TEMP anomalies; see text for details.

(a) TAUX $\mid 7.7 y r$

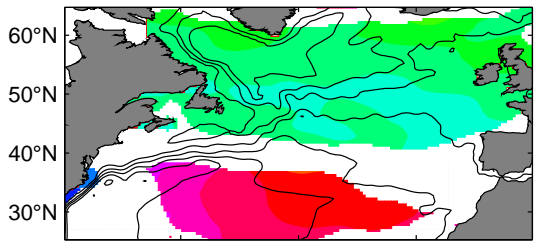

(d) TAUX | 2.7yr

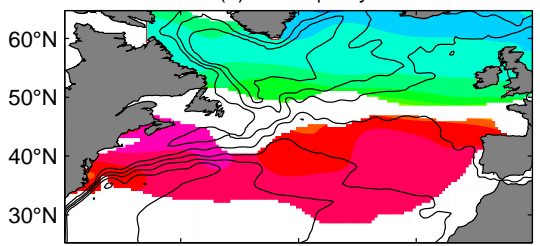

(g) TAUX | 2.2yr

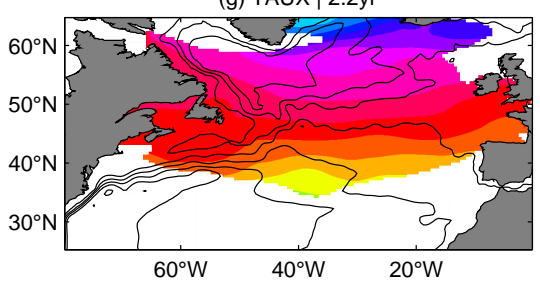

(b) $\mathrm{SSH} \mid 7.7 \mathrm{yr}$

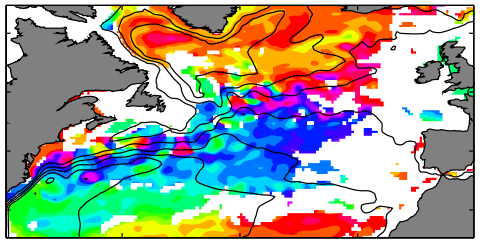

(e) $\mathrm{SSH}$ | 2.7yr

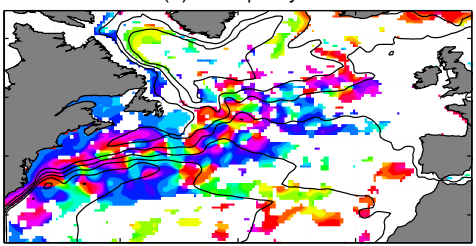

(h) $\mathrm{SSH} \mid 2.2 \mathrm{yr}$

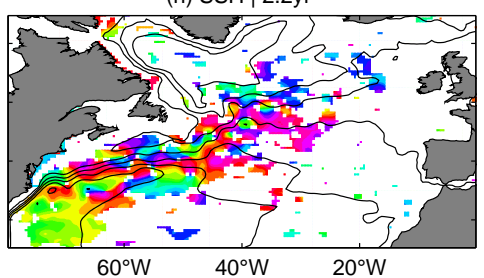

(c) SST | 7.7yr

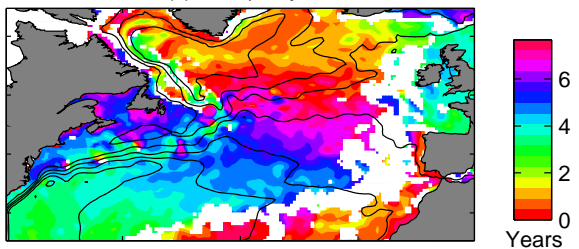

(f) SST | 2.7yr

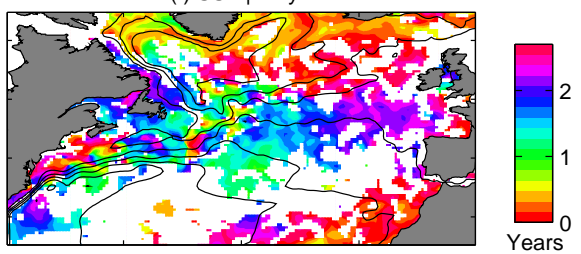

(i) SST | 2.2yr

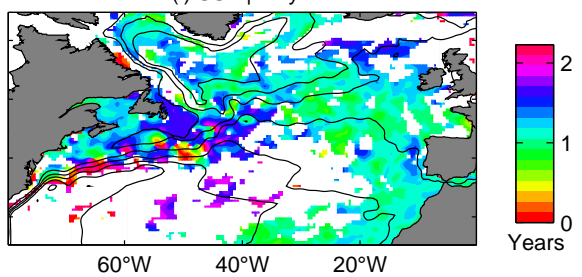

FIG. 7. Phase maps for the TAUX, SSH, and SST anomalies in the three oscillatory modes with period (a-c) $7.7 \mathrm{yr}$, (d-f) $2.7 \mathrm{yr}$, and $(\mathrm{g}-\mathrm{i})$ $2.2 \mathrm{yr}$. The color coding reflects the cyclical character of the phase, with the interval $[0,2 \pi]$ being mapped onto each of the three periods $[0, T]$, with $T=7.7,2.7$ and $2.2 \mathrm{yr}$, respectively. M-SSA parameters as in Fig. 4; grid points of low variance are masked, and the SSH CI $=0.2 \mathrm{~m}$, as before. 
(a) $7.7 \mathrm{yr} \mid$ mean $=0.82$ | sig $=0.64$

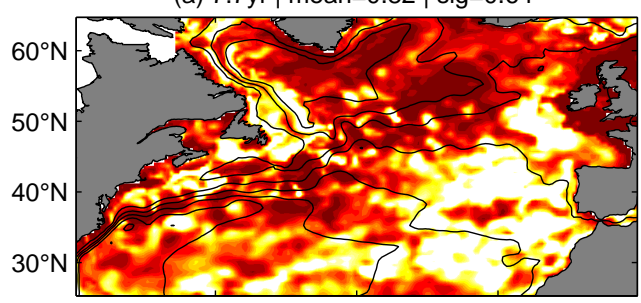

(b) $2.7 \mathrm{yr} \mid$ mean $=0.73 \mathrm{|} \mathrm{sig}=0.62$

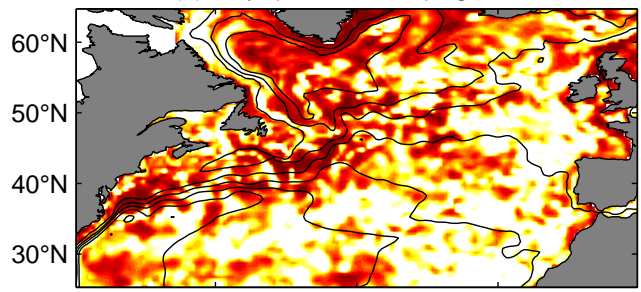

(c) $2.2 \mathrm{yr} \mid$ mean $=0.70 \mid \mathrm{sig}=0.68$

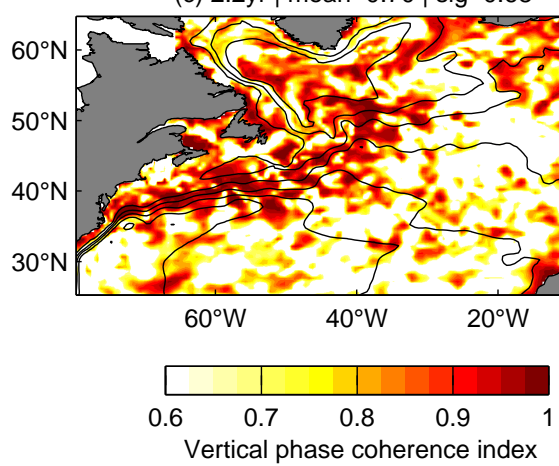

FIG. 8. Vertical phase coherence in the TEMP anomalies of the oscillatory modes with periods of (a) $7.7 \mathrm{yr}$, (b) $2.7 \mathrm{yr}$, and (c) $2.2 \mathrm{yr}$. The mean phase-coherence index (mean) is indicated in the legend, as well as a $99 \%$ level of significance (sig), against the null hypothesis of randomly shuffled phases. M-SSA parameters as in Fig. 4 , and CI $=0.2 \mathrm{~m}$ for the SSH contours (solid black).

varying patterns of intraseasonal variability with this 9month period along the front, specifically near the Gulf Stream's separation point.

In the present analysis, we concentrate instead on covariations with longer, interannual time scales, which also extend over longer, basin-wide scales, off the Gulf Stream front. To better understand dynamical properties that could underly the observed oscillatory modes, we include all available TEMP layers at greater depths as well. The vertical coherence of the observed SST pattern is then quantified in each of the modes in Fig. 7 by calculating a phase-coherence index; see Eq. (C5) in appendix C. In regions of high phase coherence, the observed SST pattern extends into deeper layers, and increasingly equivalentbarotropic behavior is thus highlighted.

Figure 8 shows the resulting maps of vertical phase coherence for the three oscillatory modes. The statistical significance of this coherence is tested against the null hypothesis of randomly shuffled phases, as derived from a random shuffling of all grid-points.

Common to all modes is a concentration of high values along the Gulf Stream front, i.e., in a region in which interannual variability is strong, cf. again Fig. 4 (left column). This finding shows that the interannual dynamics in the Gulf Stream region is characterized by a strong barotropic component, as found by Schmeits and Dijkstra (2000) for their 9-month mode near the Gulf Stream separation point.

Off the Gulf Stream front, we observe likewise high phase-coherence values, especially prominent in the 7.7yr mode (panel a). It is remarkable that the region of high phase-coherence values coincides fairly well with the area in which we see strong covariation between the SSH and SST fields; cf. again Fig. 7b,c. We are thus led to the conclusion that the SW-NE SST propagation in this 7.7yr mode is characterized by a strong equivalent barotropic component. Interestingly, we see that phase coherence is especially high in the subpolar gyre, in which we observe a cooling-like effect of the surface wind stress on SST; see Figs. 5 and 6.

In the 2.7-yr and 2.2-yr modes, on the other hand, phase-coherent behavior is much less pronounced in regions off the Gulf Stream front, cf. Figs. 8b and c, respectively. This finding is consistent with the more complex phase dynamics in these modes and the apparent absence of covariation in SSH and SST, cf. again Fig. 7, middle and lower panels.

\section{Testing the hypothesis of an ocean response to atmo- spheric forcing}

\section{a. Methodological considerations}

We address next the question to which extent the interannual oscillatory modes found in the North Atlantic TEMP field can be attributed entirely to its response to interannual variability in the atmospheric forcing. The alternative hypotheses have already been listed in this paper before - they include the ocean's intrinsic dynamics and coupled atmosphere-ocean modes. Various mechanisms for intrinsic variability have been proposed, such as relaxation oscillations of the Gulf Stream system in the presence of time-independent wind forcing (e.g., Simonnet et al. 2005; Dijkstra and Ghil 2005, and references therein) or the turbulent oscillator of (Berloff et al. 2007, and references therein); see also Liu (2012) for a historical overview of decadal to interdecadal variability.

In section 3, several oscillatory modes of similar period have been identified in separate M-SSA analyses of the TAUX, SSH and TEMP anomalies: a 7-8-yr mode and two quasi-biennial modes, with periodicities of $2.7 \mathrm{yr}$ and $2.2 \mathrm{yr}$. In section 4 , these modes have been shown, through a joint M-SSA analysis, to be consistent, in certain regions at least, with an oceanic response to atmospheric forcing. 


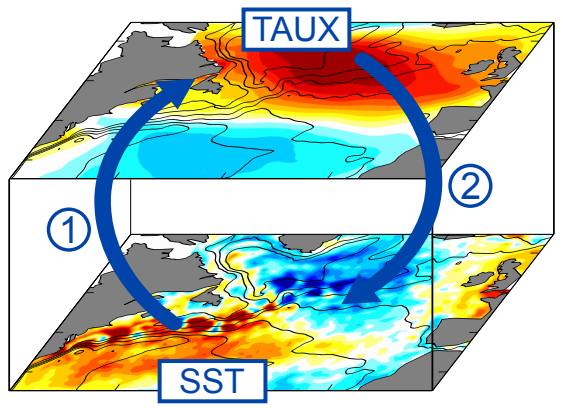

FIG. 9. Schematic diagram of interannual variability in SST and TAUX. The SST map (lower level of the figure) and the TAUX map (upper level) show phase \#3 from the phase composite analyses in Figs. 5 and 6 , respectively.

However, despite the good match in period of the oscillatory modes, the details of their temporal behavior do exhibit certain differences. In the 7.7-yr mode, for example, we observe a constant decline in amplitude in the TAUX anomalies, cf. Fig. 2d, whereas in the SSH-TEMP anomalies, the amplitude remains fairly constant over the entire 138-yr interval, cf. Fig. 3d.

The damping of this TAUX mode in time is consistent with the similar decline of the 7.7-yr mode in the TAUY anomalies and in the sea level pressure anomalies, as ascertained from the 20CRv2 reanalysis dataset (not shown). This fall-off in 7.7-yr activity in atmospheric forcing is also reflected in instrumental data, such as the NAO index (Feliks et al. 2013).

The schematic picture in Fig. 9 is meant to illustrate the mutual effects of each fluid medium on the other, as inferred here from the SODA reanalysis. In the SODA setup, the atmosphere from the $20 \mathrm{CRv} 2$ reanalysis is merely a prescribed, time-dependent boundary condition acting on the ocean model; i.e., path 1 in Fig. 9 is missing and with it the possibility of a positive feedback loop that could provide a truly coupled mechanism. This absence of path 1 might be one of the reasons for the differences we noted in the temporal evolution of interannual variability in SST vs. that in TAUX.

To overcome the limitations of a joint M-SSA analysis that may overemphasize coupled ocean-atmosphere modes, we proceed next to apply a different technique in comparing oscillatory modes in TEMP with those in TAUX. The starting point is that of separate M-SSA analyses, similar to the one in section 3 . This time, however, we also analyze the TEMP and SSH anomalies separately and not jointly, as in section 3c; i.e., we carry out three separate M-SSA analyses of (1) the TAUX anomalies, (2) the SSH anomalies, and (3) the TEMP anomalies.

Next, the M-SSA results of the TEMP anomalies are compared separately with those of the SSH and TAUX anomalies, respectively. This protocol is related to the Monte Carlo-type null-hypothesis test applied in the present paper, a test for which Groth and Ghil (2015) have reviewed several mathematical techniques. We rely here on a comparison of the time EOFs (T-EOFs), which describe the temporal pattern of the oscillatory modes; for more mathematical details see appendix D.

This way, we get two different views of the TEMP anomalies, one that is linked to the spatio-temporal dynamics of the wind stress TAUX, and the other one linked to the dynamics of the SSH field. These two views give the possibility to identify regions in the TEMP anomalies that are more likely to be dynamically linked to either the TAUX or the SSH dynamics, respectively, but that cannot be distinguished from each other by a joint M-SSA analysis of all three fields.

On the one hand, projecting the TEMP anomalies onto the TAUX anomalies highlights regions in which there is a strong association between interannual variability in the wind forcing and in the ocean temperature field. On the other, projecting TEMP on SSH is likely to reflect other, possibly intrinsic ocean dynamics.

The latter inference stands to reason, since the SODA assimilation scheme mainly corrects for differences in SST and thus has no explicit effect on the SSH field. The corrections act only implicitly on SSH, as it is linked to the vertically integrated temperature. Hence, examining closely the separate TAUX, SSH and TEMP analyses, along with the partial joint analyses of the TAUX + TEMP fields and of the SSH + TEMP fields can help answer the question of whether there is interannual variability in TEMP that cannot be explained by variations in TAUX alone.

\section{b. Overall results}

Figure 10 shows differences in the participation index $\pi$ at each grid point in the TEMP anomalies between their projection onto TAUX and SSH, respectively; see Eq. (D4) for a definition of the index $\pi$. The differences are plotted for layers at different depths in the three oscillatory modes of period $7.7 \mathrm{yr}, 2.7 \mathrm{yr}$, and $2.2 \mathrm{yr}$, respectively.

It turns out that in all nine panels, we obtain a mixture of positive and negative differences, i.e., a stronger link of the TEMP anomalies to SSH or TAUX, respectively. These differences are comparable in absolute value to the amplitude of the three oscillatory modes, cf. Fig. 4, and it appears that positive and negative differences are geographically well structured and not just randomly distributed. This systematic character of the differences points to the existence of mechanisms that yield a stronger link of TEMP to either SSH or TAUX over well-defined regions.

Common to all oscillatory modes in Fig. 10, we observe at the surface (left column) a concentration of small spots of positive differences along the Gulf Stream front. This 
(a) $7.7 \mathrm{yr}|5 \mathrm{~m}| \min =-0.18, \max =0.23$

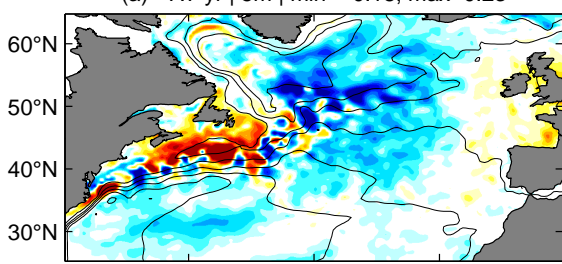

(d) $2.7 \mathrm{yr}|5 \mathrm{~m}| \min =-0.46, \max =0.29$

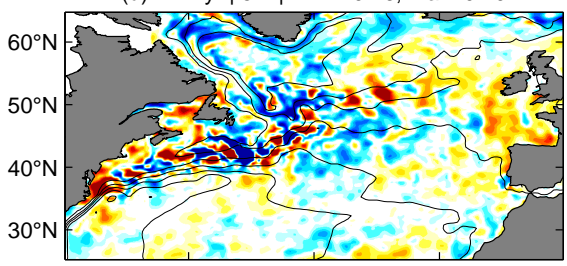

(g) $2.2 \mathrm{yr}|5 \mathrm{~m}| \mathrm{min}=-0.28, \max =0.34$

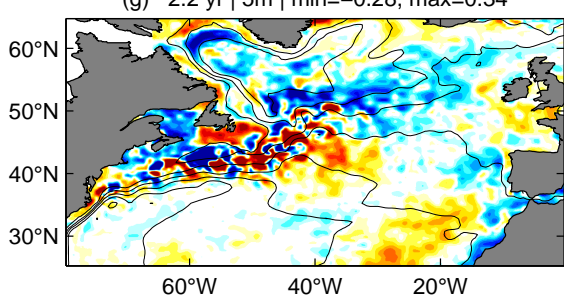

(b) $7.7 \mathrm{yr}|171 \mathrm{~m}| \min =-0.26, \max =0.29$

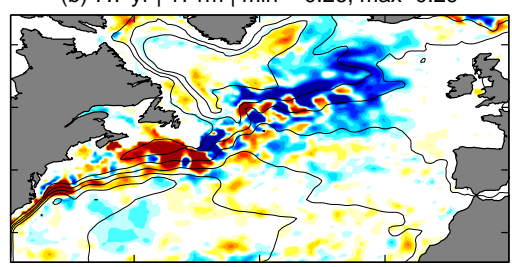

(e) $2.7 \mathrm{yr}|171 \mathrm{~m}| \min =-0.35$, $\max =0.52$

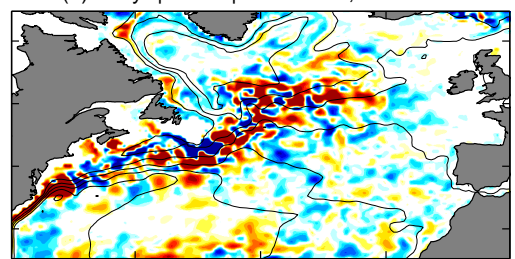

(h) $2.2 \mathrm{yr}|171 \mathrm{~m}| \min =-0.26, \max =0.40$

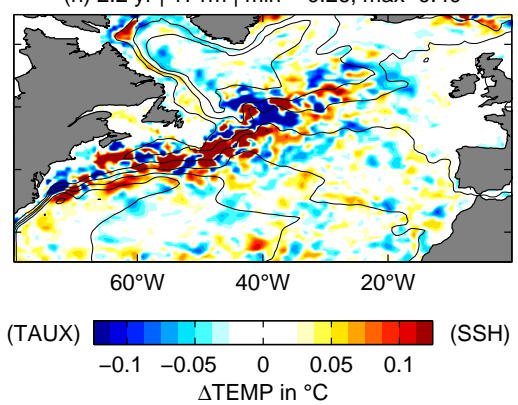

(c) $7.7 \mathrm{yr}|466 \mathrm{~m}| \min =-0.18, \max =0.33$

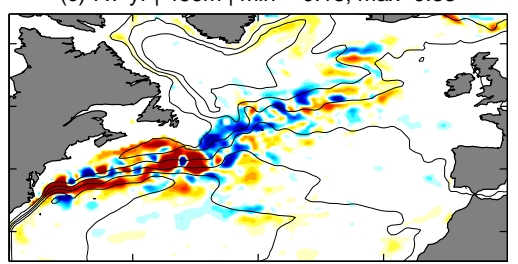

(f) $2.7 \mathrm{yr}|466 \mathrm{~m}| \min =-0.33$, $\max =0.55$

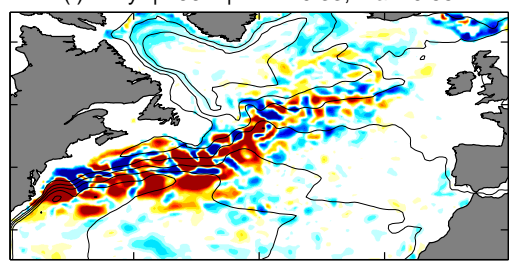

(i) $2.2 \mathrm{yr}|466 \mathrm{~m}| \min =-0.25$, $\max =0.33$

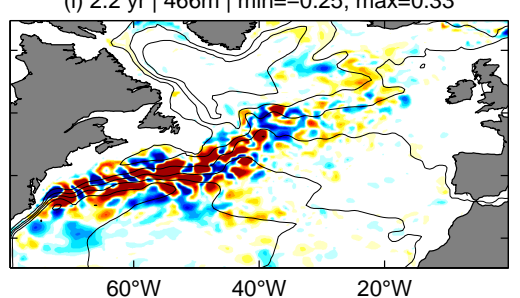

FIG. 10. Differences in the participation index $\pi$ (in color) between a projection of the TEMP anomalies onto the SSH anomalies and onto the TAUX anomalies, respectively. Shown are the square root of the differences in (a-c) the 7.7-yr mode, (d-f) the 2.7-yr mode, and (g-i) the 2.2-yr mode, at the depths of (a,d,g) $5 \mathrm{~m}$, (b,e,h) $171 \mathrm{~m}$, and (c,f,i) $466 \mathrm{~m}$. Extreme values are given in the panel legends (min and max), in degrees $\mathrm{K}$. M-SSA parameters for TAUX and SSH as in Figs. 2 and 3, respectively. SSH CI = $0.2 \mathrm{~m}$.

concentration indicates a stronger link between the spectral properties of the TEMP and SSH anomalies in the Gulf Stream region.

It is striking that this link becomes stronger with increasing depth (middle and right column); i.e., the spots of positive differences increase in size and absolute value with depth, as shown also by the increase in maximum value (max) in the panel legends. The persistence and even enhancement of SSH covariance with ocean temperatures at depth is to some extent expected, as we have already identified strongly phase-coherent behavior in this region, cf. Fig. 8.

It is plausible to attribute such a strong link between TEMP and SSH to equivalent-barotropic behavior in this region and at long periods. Equivalent-barotropic behavior, in turn, could also point to the existence of intrinsic variability along the Gulf Stream front, as predicted, for example, in the setting of gyre-mode instability, in which barotropic gyre modes emerge on interannual time scales.

Beside the strong link between TEMP and SSH in all three oscillatory modes along the Gulf Stream front, we observe in Fig. 10 regions off the Gulf Stream front that show negative differences, i.e. in which interannual vari- ability in TEMP is strongly linked to the TAUX variability. In particular, in the 7.7-yr mode at the surface (panel a), there is an area of strong negative differences in the subpolar gyre, which coincides with the region of maximum variability in TAUX, cf. Fig. 4a. This region is remarkably consistent with the one in which we have already identified a cooling effect.

According to Figs. 10b,c, the area of negative differences gradually vanishes with depth, as one might expect for a surface effect. At the surface (panel a), we also observe smaller negative values that cover a large region off the Gulf Stream front, in which we have observed a southwest-to-northeast propagating wave. This finding suggests an ocean response in SST due to variations in TAUX.

For the 2.7-yr mode, the middle panels of Fig. 10 show a qualitatively different picture. In all three layers, the mix of positive and negative differences and the association with the atmospheric forcing become less clear. Specifically, negative values are present in many regions of the North Atlantic and they are not limited to regions of strong TAUX variability in this mode, cf. Fig. 4d. Still, a tendency of generally more negative values at the surface and 
gradually more positive values along the Gulf Stream front at greater depth becomes apparent in Fig. 10; see also the min and max values in the panel legends.

The picture in the 2.2-yr mode (Fig. 10, lower panels) resembles qualitatively that of the 7.7-yr mode; i.e., negative values prevail at the sea surface where TAUX variations are strong, cf. Fig. 4g, and where TEMP and TAUX anomalies are in phase opposition, cf. Figs. 7f,g. The influence of TAUX variations acting on the ocean's TEMP fields cf. Figs. 10h,i, likewise seems to vanish with increasing depth, where at the same time gradually more positive values along the Gulf Stream front emerge and indicate an increasingly stronger link with SSH.

Although the present analysis alone cannot provide definitive conclusions about the underlying dynamics, it is consistent with the view that interannual variability in the North Atlantic basin cannot be explained by the ocean's response to similar variability in the atmospheric forcing alone (e.g., Dijkstra and Ghil 2005; Berloff et al. 2007; Penduff et al. 2011; Sérazin et al. 2015).

\section{c. Changing patterns in the 7.7-yr mode}

In this subsection, we are interested in a better understanding of the declining amplitude in the 7.7-yr mode in the TAUX anomalies, cf. Fig. 2d. The passive-ocean point of view would suggest a similar damping of the corresponding oscillatory pattern in the SST anomalies.

We thus divide the 138-yr SODA dataset into two shorter time intervals, (i) 1871-1950 and (ii) 1929-2008, respectively. The small, 20 -yr overlap is necessary insofar as we keep a window length of $M=40 \mathrm{yr}$, and therefore need a minimal length of $N \geq 2 M=80 \mathrm{yr}$ for each subinterval. In each of the two segments, we then run a joint M-SSA analysis of the TAUX, SSH, and TEMP anomalies, with the same parameters as in section 4 .

In the first, 1871-1950 segment, we obtain a 7-8-yr mode (not shown) that resembles in many of its spatiotemporal details to the 7.7-yr mode in the full 138 -yr interval, as seen in section 4 , and in the supplementary animation.

The picture changes, however, when we analyze the second, 1929-2008 segment; see Figs. 11 and 12. There is still a 7-8-yr oscillatory mode with a similar period, but the corresponding phase-composite analysis reveals different dynamics in both TAUX and SST. In the phase composites in the TAUX anomalies in Fig 11, we observe a shift in the geographical extension, with the maximum now displaced further east. An analysis of TAUY (not shown separately, but see arrows in the figure) indicates that the decline in TAUX is not compensated by an increase in TAUY, and that TAUX still plays a dominate role in the wind stress.
In the corresponding phase composites of the SST anomalies (Fig. 12), this eastward displacement of the stationary pattern is accompanied by a vanishing of the SWNE propagating pattern previously seen in Fig. 5. Remarkably, though, the interannual variability along the Gulf Stream front remains quite high in Fig. 12, if not even higher in amplitude than in the 7.7-yr mode of the full dataset in Fig. 5.

In our separate M-SSA analysis of the TAUX anomalies alone for 1929-2008, it turns out that the 7-8-yr period of interannual variability is no longer significant (not shown). This finding is consistent with section 3 , in which we found the 7.7-yr mode significant in TAUX in the first, 1871-1950 segment only.

The overall agreement between basinwide changes in the atmospheric TAUX and in the oceanic SST fields leads us to the conclusion that the observed traveling wave with a 7-8-yr periodicity off the Gulf Stream can, indeed, be attributed to the ocean's response to interannual variability in the atmospheric forcing. In the Gulf Stream region, though, this change shows that 7-8 year variability cannot merely be attributed to the atmospheric forcing; see also the concluding discussion in section $6 \mathrm{~b}$ below about the possibility of intrinsic ocean dynamics.

The cause of the changes in the atmospheric forcing pattern, however, remains unclear from the present analysis. The changes themselves, though, appear to be consistent with the observed decadal fluctuations noted by Deser and Blackmon (1993). Moreover, we are able to identify a similar change (not shown) in the SST dataset of Kaplan et al. (1998).

\section{Concluding remarks}

\section{a. Summary}

By analyzing spectrally the evolution in time of the North Atlantic temperature field, denoted here by TEMP, in the Simple Ocean Data Analysis (SODA) reanalysis (Carton and Giese 2008; Giese and Ray 2011), we were able to identify and describe prominent and statistically significant interannual oscillations along the Gulf Stream front and in large regions of the North Atlantic. The extensive reanalysis dataset has been decomposed into different patterns of spatio-temporal variability with the advanced spectral method of multivariate singular spectrum analysis (M-SSA); see Ghil et al. (2002) and Alessio (2016) for a comprehensive review of M-SSA, and Groth and Ghil (2011, 2015) for recent improvements thereof that help separate distinct spatio-temporal patterns.

The M-SSA results on the temperature field were compared with the M-SSA results on the zonal wind stress forcing, denoted by TAUX, from the atmospheric 20CRv2 reanalysis (Compo et al. 2011). In the interannual frequency band, three oscillatory modes with periods of 

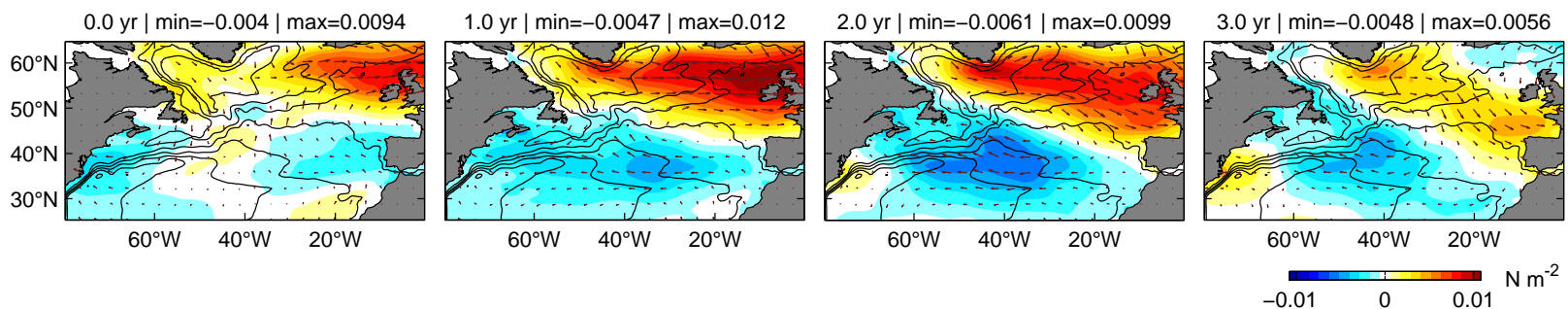

FIG. 11. Phase composites in TAUX (in color) from the 7-8-yr oscillatory mode of a joint M-SSA analysis of TAUX, SSH, and TEMP anomalies, during the second, 1929-2008 subinterval; see text for details. Four phases that correspond to one half of the cycle are shown. The phase categories are labeled in the panel legends by their midpoint (in years); also shown are extreme values (min and max). Superimposed phase composites of the vector wind stress (arrows) are reconstructed from a projection of the TAUY anomalies onto the joint M-SSA analysis of the TAUX, SSH, and TEMP anomalies. M-SSA parameters as in Fig. 4; SSH CI $=0.2 \mathrm{~m}$.

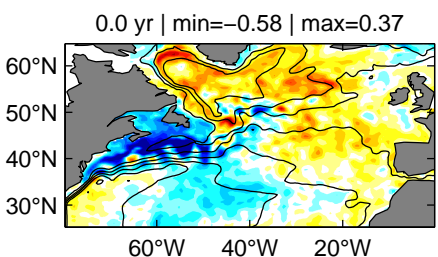

$60^{\circ} \mathrm{W} \quad 40^{\circ} \mathrm{W} \quad 20^{\circ} \mathrm{W}$

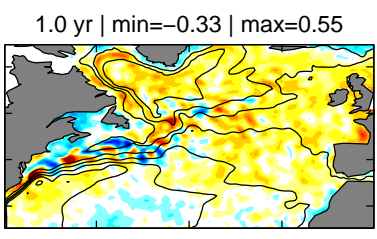

$60^{\circ} \mathrm{W} \quad 40^{\circ} \mathrm{W} \quad 20^{\circ} \mathrm{W}$

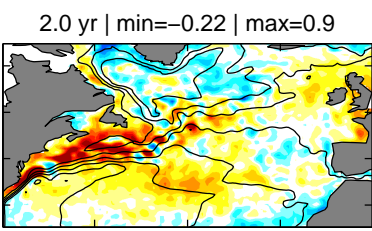

$60^{\circ} \mathrm{W} \quad 40^{\circ} \mathrm{W} \quad 20^{\circ} \mathrm{W}$

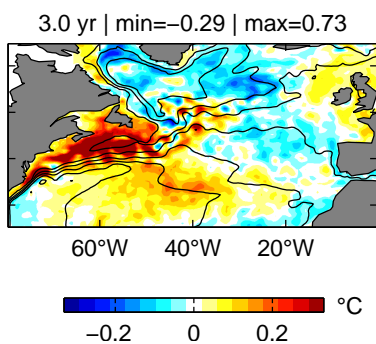

FIG. 12. Same as Fig. 11, but in the SST anomalies.

$7.7 \mathrm{yr}, 2.7 \mathrm{yr}$, and $2.2 \mathrm{yr}$ and high statistical significance that are similar in both TAUX and TEMP were identified, cf. section 3. These findings are consistent with those of numerous other publications that studied interannual variability in the atmospheric field over the North Atlantic (e.g., Rogers 1984; Hurrell 1995; Hurrell and Van Loon 1997; Robertson 2001; Feliks et al. 2010), and in the North Atlantic SSTs (e.g., Moron et al. 1998; Da Costa and Colin de Verdière 2002; Jamison and Kravtsov 2010).

To understand patterns of interannual variability in the coupled ocean-atmosphere system, these three modes were furthermore analyzed in a joint M-SSA analysis, cf. section 4. It turned out that the 7.7-yr oscillatory mode is characterized by a basin-wide southwest-to-northeastoriented propagation pattern in SSTs.

This SST pattern is similar to the one identified in several previous papers (Sutton and Allen 1997; Da Costa and Colin de Verdière 2002; Simonnet et al. 2005; Jamison and Kravtsov 2010) and it was shown here to be linked to alternations in the meridional dipole pattern in TAUX. In the subpolar gyre, the SST and TAUX fields are in phase opposition, which indicates a cooling effect of the wind stress on the upper ocean layer, in agreement with Deser and Blackmon (1993) and Sutton and Allen (1997); see also the supplementary animation herein. In our analysis, this propagating SST pattern is characterized, furthermore, by a strong equivalent-barotropic component, which leads to covariations in the SST and SSH fields, and phasecoherent behavior of the observed SST pattern with the temperature layers in the deep ocean that are available in the TEMP field of the SODA reanalysis.

In the two modes of quasi-biennial variability, though, the dynamics turned out to be more complex: There is no clear indication of covariation between SST and SSH off the Gulf Stream region, and the association with the wind stress forcing is less obvious.

Finally, in section 5, we analyzed to which extent the observed patterns of interannual variability in the North Atlantic temperature field can be attributed exclusively to variations in the atmospheric forcing (Bjerknes 1964; Hasselmann 1976), as opposed to or in combination with intrinsic ocean dynamics. We have shown that in large regions off the Gulf Stream front, the wind stress forcing has a substantial influence on the interannual variability of the underlying ocean in all three modes - namely the 7.7-yr mode and the two quasi-biennial modes - but also that this influence gradually decreases with depth.

Along the Gulf Stream front, our analysis supports the existence of other, internal mechanisms in all three modes; furthermore, the influence of the latter mechanisms on interannual variability increases with depth. Differences in the oscillatory modes between the wind stress forcing and the ocean temperature field were most prominent in the 7-8-yr mode: we observe a pronounced decline in amplitude of the atmospheric forcing, while the amplitude 
of this mode in the ocean dynamics is quite constant, cf. Figs. 2c and 3c. This decline in amplitude is accompanied by a change in the spatial pattern of the atmospheric forcing in the latter part of the dataset, i.e. 1929-2008, cf. Fig. 12, as well as in the SST pattern.

In fact, the SW-NE SST propagation across the North Atlantic basin disappears in the latter time interval, while 7-8-yr variability along the Gulf Stream front persists. This persistence is quite remarkable and it supports even more strongly the idea that variability in the 7-8-yr frequency band in the Gulf Stream region is substantially influenced by other mechanisms, and not merely a response to 7-8-yr variability in the atmospheric forcing. Our findings in this respect are consistent with the view that interannual variability in the North Atlantic basin cannot be explained by the ocean's response to similar variability in the atmospheric forcing alone.

\section{b. Discussion}

The M-SSA analyses of sections 3-5 suggest that different types of mechanisms participate in the variability of the oceanic TEMP field; these mechanisms are well characterized by their distinct spatio-temporal patterns.

On the one hand, our analysis of the SODA dataset shows the substantial influence that midlatitude atmospheric perturbations exert on the underlying ocean, away from the regions of intense oceanic currents, (cf. Frankignoul and Hasselmann 1977; Frankignoul et al. 1997; Sérazin et al. 2015), and that large-scale interannual variability in the atmospheric forcing is indeed reflected in the underlying ocean's large-scale SST response (Bjerknes 1964); see again Fig. 9 here, in which this influence is schematically indicated as path 2 . Several studies of ocean-atmosphere interaction over the North Atlantic basin have highlighted the role of an SST tripole pattern (e.g., Czaja and Frankignoul 1999; Marshall et al. 2001; Czaja and Frankignoul 2002; Czaja et al. 2003; Kravtsov et al. 2008; Jamison and Kravtsov 2010). This large-scale pattern (not shown) resembles to some extent that of our 7.7-yr mode. The latter, though, involves SW-to-NE propagation that connects the pole in the Gulf Stream region with the one in the subpolar gyre. To which extent these two patterns describe the same physical mechanism is left for future studies.

On the other hand, there is strong variability in highly localized spots along the Gulf Stream front, which can be plausibly linked to internal ocean dynamics; see also Penduff et al. (2011) and Sérazin et al. (2015). The present analysis does not ascertain the extent to which this oceanic variability emerges through gyre-mode instabilities and homoclinic bifurcations or from the turbulent oscillator of Berloff et al. (2007, and references therein).
Still, the statistical evidence of equivalent-barotropic behavior in this region is entirely consistent with the gyremode hypothesis, such as confirmed by several studies with more or less idealized double-gyre ocean models (Jiang et al. 1995; Speich et al. 1995; Simonnet and Dijkstra 2002; Simonnet et al. 2003a,b, 2005; Sushama et al. 2007). Across a hierarchy of models - from simple rectangular-shaped ocean basins and 1.5-layer reducedgravity models (Jiang et al. 1995; Speich et al. 1995; Chang et al. 2001; Simonnet et al. 2003a) to more realistic North Atlantic-basin shapes, several layers and eddypermitting resolutions (Simonnet et al. 2003b, 2005) - it was found that the idealized double-gyre flow becomes unstable to two types of barotropic modes: basin modes and gyre modes. The time scales of the former are a couple of months (Chang et al. 2001), whereas the latter instabilities have interannual time scales of 7-8 yr in the North Atlantic (Speich et al. 1995; Simonnet et al. 2005), similar to the one identified here in the SODA reanalysis. On the other hand, the turbulent-oscillator hypothesis depends in an essential way on the vigorous activity of baroclinic eddies.

Whatever the underlying mechanisms of interannual variability, it is interesting to note that, in a series of increasingly detailed atmospheric models, Feliks et al. (2004, 2007, 2011, 2016), Brachet et al. (2012) and Deremble et al. (2012) have shown that sharp SST gradients, like the Gulf Stream or Kuroshio fronts, can have a strong influence on the overlying atmosphere and its lowfrequency variability. Variations in frontal strength, for example, were shown to modulate the atmospheric jet's intensity; see also Schneider and Qiu (2015) and Kilpatrick et al. (2016, and references therein) for details on the Ekman pumping mechanism that mediates the SST front effect on the midlatitude atmosphere.

This modeling result on the atmospheric effects of ocean fronts (Small et al. 2008) is consistent with statistical evidence (Mosedale et al. 2006) for an SST effect, especially south of Cape Hatteras, on the NAO; see again Fig. 9 here, in which this potential spin-up mechanism for atmospheric interannual variability is indicated by path 1 . Thus, one might be able to anticipate low-frequency fluctuations in the atmosphere above and downstream of the North Atlantic basin by monitoring of the Gulf Stream.

Such monitoring could involve its total transport across a meridional section off Cape Hatteras — as done systematically by the Japan Meteorological Agency for the Kuroshio (Imawaki et al. 2001) — as well as the SST and SSH gradients across such a section.

Acknowledgments. It is a pleasure to acknowledge stimulating discussions with J. C. McWilliams. The authors would also like to thank three anonymous reviewers for their extremely careful and constructive reviews. 
The research herein was supported by grants N00014-121-0911 and N00014-16-1-2073 from the Multidisciplinary University Research Initiative (MURI) of the Office of Naval Research and by National Science Foundation grant OCE-1243175.

\section{APPENDIX A}

\section{Singular spectrum analysis}

In this appendix, we briefly summarize the M-SSA algorithm as originally proposed by Broomhead and King (1986a,b). For a review of the full M-SSA methodology, as used herein, see Ghil et al. (2002), Groth and Ghil (2011, 2015), Alessio (2016) and references therein.

Let $\mathbf{x}=\left\{x_{d}(n): d=1, \ldots, D, n=1, \ldots, N\right\}$ be a multivariate time series with $D$ channels of length $N$. Each channel is embedded into an $M$-dimensional phase space by using lagged copies $\mathbf{X}_{d}(n)=\left[x_{d}(n), \ldots, x_{d}(n+M-1)\right]$, $n=1, \ldots, N-M+1$, from which we form the full augmented trajectory matrix by concatenating all channels, $\mathbf{X}=\left(\mathbf{X}_{1}, \mathbf{X}_{2}, \ldots, \mathbf{X}_{D}\right)$. The matrix $\mathbf{X}$ has $D M$ columns of length $N^{\prime}=N-M+1$.

The M-SSA algorithm then continues with a singular value decomposition (SVD) of $\mathbf{X}$,

$$
\mathbf{X}=\eta^{1 / 2} \mathbf{P} \Sigma \mathrm{E}^{\prime},
$$

where $\eta$ is a normalization factor equal to the larger of $N^{\prime}$ and $D M$. The matrix $\boldsymbol{\Sigma}$ has $\kappa$ non-vanishing diagonal elements, the singular values $\left\{s_{k}: k=1, \ldots, \kappa\right\}$, where $\kappa$ is equal to the smaller one of $N^{\prime}$ and $D M$. The singular vectors $\mathbf{P}$ and $\mathbf{E}$ have both rank $\kappa$. The left-singular vectors $\mathbf{P}$ describe pure temporal behavior, where the right-singular vectors $\mathbf{E}$ describes spatio-temporal behavior; i.e. $\mathbf{P}$ is referred to as time EOFs (T-EOFs), and $\mathbf{E}$ as space-time EOFs (ST-EOFs). The latter are composed of $D$ consecutive segments $e_{d k}$ of length $M$, each of which is associated with a channel in $\mathrm{X}_{d}$ given by $e_{d k}(m)=\left[e_{k}[(d-1) M+m]\right.$.

A projection of $\mathbf{X}$ onto $\mathbf{E}$,

$$
A=X E,
$$

gives a set of $\kappa$ pairwise uncorrelated principal components (PCs), of reduced length $N^{\prime}$.

Dynamical behavior that is associated with a subset $\mathscr{K} \subseteq\{1, \ldots, \kappa\}$ of ST-EOFs can be reconstructed from

$$
\mathrm{R}_{\mathscr{K}}=\mathrm{AKE}^{\prime} \equiv \mathrm{XEKE^{ \prime }},
$$

with $\mathrm{K}$ a diagonal matrix of size $\kappa \times \kappa$, in which elements $K_{k k}=1$ if $k \in \mathscr{K}$ and $K_{k k}=0$ otherwise.

Upon averaging along the skew diagonals of $\mathbf{R}$,

$$
r_{d \mathscr{K}}(n)=\frac{1}{M_{n}} \sum_{k \in \mathscr{K}} \sum_{m=L_{n}}^{U_{n}} a_{k}(n-m+1) e_{d k}(m),
$$

we finally obtain the reconstructed components (RCs). The normalization factor $M_{n}$ and the summation bounds $L_{n}$ and $U_{n}$ for the central part of the time series, $M \leq n \leq$ $N-M+1$, are simply $\left(M_{n}, L_{n}, U_{n}\right)=(M, 1, M)$. For either end of the series, $1 \leq n<M$ or $N-M+1<n \leq N$, these values are modified according to the length of the skew diagonal corresponding to the given $n$; see, for instance, Ghil et al. (2002).

In the application at hand, $N=138$ and the values of $D$ for the multivariate time series analyzed in sections 35 are of the order of $10^{5}$ for the two-dimensional arrays TAUX, SSH and SST, and of the order of $10^{6}$ for the threedimensional array TEMP, as well as for those involved in the joint M-SSA analyses that include TEMP.

\section{APPENDIX B}

\section{Projection onto spatial EOFs}

The large values of $D$ used herein are typical nowadays in the climate sciences, whether one studies recent, instrumental datasets from both in situ and satelite-borne observing systems or long climate simulations by high-end models (Solomon et al. 2007; Stocker et al. 2013). We discuss, therefore, now the implications of projecting a large dataset, i.e. $D \gg N$, onto classical S-EOFs.

Prior to the M-SSA analysis, the multivariate time series $\mathbf{x}$ is first transformed into a set of $L=N$ classical PCs via a SVD, $\mathbf{x}=\mathbf{u s v}^{\prime}$, and the PCs, $\mathbf{y}=\mathbf{x v}$, as input channels for M-SSA.

As in the previous section, M-SSA starts with embedding each channel in $\mathbf{y}$ into an $M$-dimensional phase space by using lagged copies $\mathbf{Y}_{d}(n)=\left[y_{d}(n), \ldots, y_{d}(n+M-1)\right]$, $n=1, \ldots, N-M+1$, from which we obtain the matrix $\mathbf{Y}=\left(\mathbf{Y}_{1}, \mathbf{Y}_{2}, \ldots, \mathbf{Y}_{L}\right)$ of size $L M \times N^{\prime}$.

It can be seen that the relation $\mathbf{y}=\mathbf{x v}$ simply translates into

$$
\mathbf{Y}=\mathbf{X}\left(\mathbf{v} \otimes \mathbf{I}_{M}\right),
$$

with $\otimes$ the Kronecker product and $\mathbf{I}_{M}$ the identity matrix of size $M$.

Next, M-SSA continues in calculating the SVD of $\mathbf{Y}$,

$$
\mathbf{Y}=\eta^{1 / 2} \mathbf{U S V}^{\prime}
$$

with the normalization factor $\eta$ as in Eq. (A1).

Replacing $\mathbf{X}$ and $\mathbf{Y}$ in (B1) by their SVDs, Eqs. (A1) and (B2), respectively, it follows that

$$
\eta^{1 / 2} \mathbf{U S V}^{\prime}=\eta^{1 / 2} \mathbf{P} \Sigma \mathbf{E}^{\prime}\left(\mathbf{v} \otimes \mathbf{I}_{M}\right) .
$$

The matrix $\mathbf{v} \otimes \mathbf{I}_{M}$ is orthogonal and so is $\mathbf{E}^{\prime}\left(\mathbf{v} \otimes \mathbf{I}_{M}\right)$.

Since the SVD of a real-valued matrix is unique up to a pairwise change in the sign of the singular vectors, it follows that $\mathbf{X}$ and $\mathbf{Y}$ have the same singular values, $\boldsymbol{S}=\boldsymbol{\Sigma}$, 
and in case of non-degenerate singular values,

$$
\begin{aligned}
\mathbf{P} & =\mathbf{U} \mathbf{T}, \\
\mathbf{E} & =\left(\mathbf{v} \otimes \mathbf{I}_{M}\right) \mathbf{V} \mathbf{T},
\end{aligned}
$$

with $\mathbf{T}$ a diagonal matrix of elements $\{-1,1\}$. That is the left- and right-singular vectors from $X$ are (up to the sign) determined by the singular vectors of $\mathbf{Y}$ and $\mathbf{v}$.

Substituting $\mathbf{E}$ in the RCs of $\mathbf{X}$, cf. Eq. (A3), according to Eq. (B4) then gives

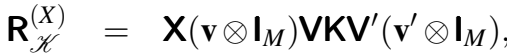

$$
\begin{aligned}
& =\mathbf{Y V K V}^{\prime}\left(\mathbf{v}^{\prime} \otimes \mathbf{I}_{M}\right) \text {. }
\end{aligned}
$$

With $\mathbf{R}_{\mathscr{K}}^{(Y)}=\mathbf{Y V K V}^{\prime}$, the RCs of $\mathbf{Y}$, it follows that

$$
\mathbf{R}_{\mathscr{K}}^{(X)}=\mathbf{R}_{\mathscr{K}}^{(Y)}\left(\mathbf{v}^{\prime} \otimes \mathbf{I}_{M}\right)
$$

Upon channel-wise averaging along skew diagonals on either side of Eq. (B6), cf. Eq. (A4), we finally obtain the relation

$$
\mathbf{r}_{\mathscr{K}}^{(X)}=\mathbf{r}_{\mathscr{K}}^{(Y)} \mathbf{v}^{\prime}
$$

between the RCs of $\mathbf{x}$ and that of $\mathbf{y}$; i.e. the Kronecker product $\mathbf{v}^{\prime} \otimes \mathbf{I}_{M}$ simplifies to $\mathbf{v}^{\prime} \otimes \mathbf{I}_{1} \equiv \mathbf{v}^{\prime}$ when returning from $X$ to $\mathbf{x}$.

Equation (B7) has important practical implications in the M-SSA analysis of extensive datasets, as it can be broken down into three smaller parts:

1. The dataset $\mathbf{x}$ is first transformed into PCs via a classical SVD analysis, i.e. $\mathbf{x}=\mathbf{u s v}^{\prime}$;

2. M-SSA ST-EOFs and RCs are determined from $\mathbf{y}=$ $\mathbf{x v}$; and

3. these ST-EOFs and RCs are then back-transformed to get the ST-EOFs and RCs of the full dataset $\mathbf{x}$ by using Eqs. (B4) and (B7), respectively.

Equation (B7) also demonstrates that there is in particular no loss of information in the RCs, when we retain the full set of $L$ PCs.

The only difference appears when we wish to rotate the ST-EOFs. Since the varimax criterion is a nonlinear function of the eigenelements, the rotation results of $\mathbf{V}$ and $\mathbf{E}$ can differ. In our experience with the SODA dataset, however, these differences are minor, and we observe likewise good results when rotating $\mathbf{V}$ instead.

To which extend a prior projection of $\mathbf{x}$ onto just a subset of S-EOFs can influence the reconstruction quality of M-SSA will be subject of the remainder in this section. We follow the simulation experiment of Groth and Ghil (2015) and first generate a noise-free realization of our reference signal to be reconstructed, see Fig. B1, upper panel. Superimposed on the reference signal is spatiotemporally correlated red noise, generated from $\mathrm{AR}(1)$
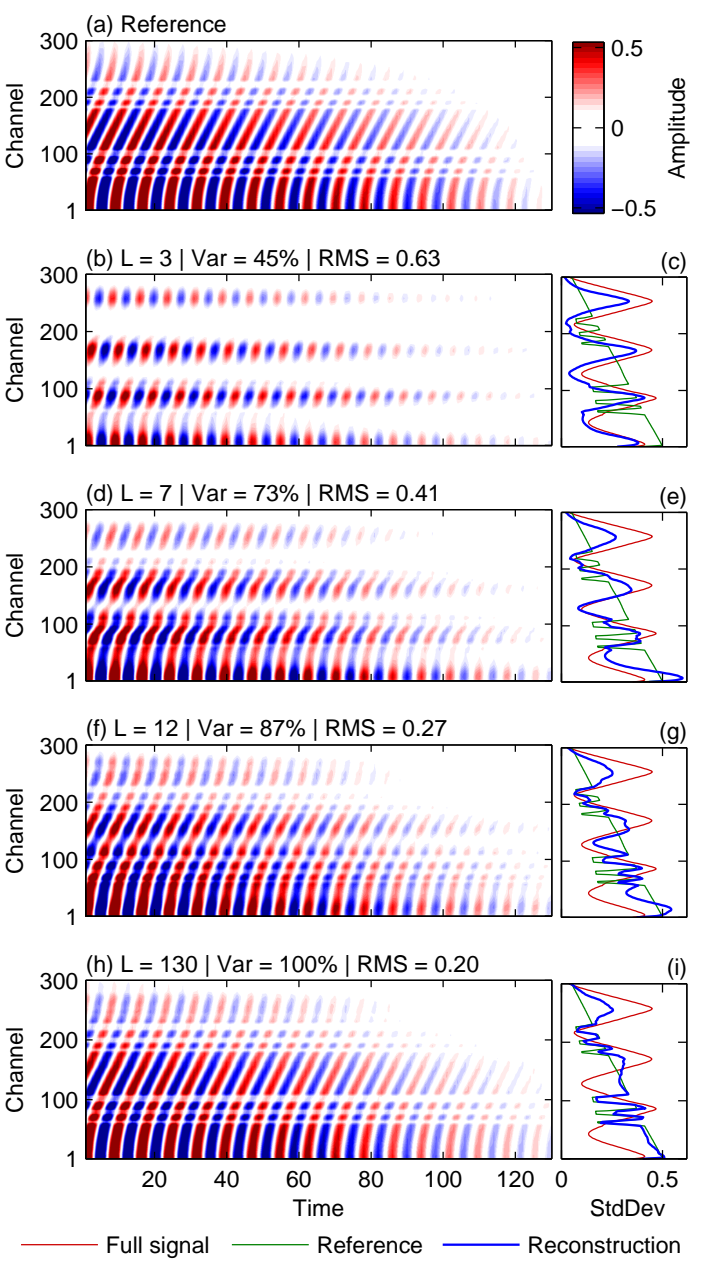

FIG. B1. Reconstruction of spatio-temporal oscillations in the presence of strong correlated noise. (a) Noise-free reference signal, $D=300$ channels of length $N=130$. (b-g) The noise-contaminated signal is first projected onto the leading $L=\{3,7,12, N\}$ S-EOFs from a conventional PCA analysis, which explains $45 \%, 73 \%, 87 \%$, and $100 \%$ of the total variance, respectively. The resulting PCs are analyzed via M-SSA with a window length $M=40$ and the first 20 ST-EOFs varimax rotated. (b,d,f,h) Average reconstruction from an ensemble of 200 repetitions of the experiment. (c,e,g,i) Corresponding channel-wise standard deviation of the full signal (red), the reference signal (green), and the average reconstruction (blue). The signal-to-noise ratio is $1 / 12$. For illustration purpose, the standard deviation of the full signal is scaled by $12^{-1 / 2}$.

processes, with a randomly drawn parameter from the interval $\gamma \in[0,0.85]$. The non-vanishing lag-0 covariance matrix $\mathbf{W}_{i j}=0.85^{|i-j|}$ of the noise, as well as its variance modulation in space, cf. the red line in Fig. B1, panel c, is meant to dominate the structure of the leading S-EOfs.

Based on an ensemble of 200 repetitions of the experiment, we evaluate statistical properties of the M-SSA reconstruction. To identify in each realization the signal part, we follow Groth and Ghil (2015) and select a pair 
of ST-EOFs that matches best the leading pair of ST-EOFs in the M-SSA analysis of the noise-free reference signal.

Figure B1, left column, shows the M-SSA reconstruction averaged over the ensemble of repetitions of the experiment for varying parameter $L$. For $L=3$ (panel b), the set of the leading three $\mathrm{S}-\mathrm{EOF}$ captures on average only $45 \%$ of the total variance. As a consequence of such a strong compression, the wave propagation in the reference signal is completely missed. Instead, the spatial structure of the reconstruction (panel c, blue line) follows that of the full signal (red line), and completely misses the linear increase and the small-scale line structure in the reference signal (green line).

As the number of S-EOFs increases, the reconstructed pattern gradually approaches that of the reference pattern. It is the projection onto the full set of S-EOFs that, finally, gives the best reconstruction quality (lower panels); i.e. it perfectly reconstructs the wave propagation. We have also analyzed the full dataset without any prior projection onto S-EOFs (not shown) and it turns out that there is no significant improvement in this particular case.

The experiment clearly illustrates the risk that a projection onto a small number of S-EOFs entails, i.e. the risk of the reconstruction being biased toward other large-scale patterns. It also shows that wave propagation patterns and small-scale structures are likely to be missed, and we recommend retaining a maximum number of PCs as input channels for the subsequent M-SSA analysis.

\section{APPENDIX C}

\section{Phases composites and phase maps}

A helpful tool for the understanding of the spatio-temporal dynamics of reconstructed oscillatory behavior is that of a phase-composite analysis, cf. Plaut and Vautard (1994) and Moron et al. (1998). Usually, given the reconstruction $\mathbf{r}$, its SVD is calculated,

$$
\mathbf{r}=\mathbf{U S V}^{\prime}
$$

and a phase is defined as the argument of the leading PC, expanded into the complex plane via either (i) its first derivative, or (ii) its Hilbert transform.

In the present work, though, we use a more direct approach, without the need of any further calculations that expands the leading PC into the complex plane. Feliks et al. (2013), for example, have shown that upon varimax rotation of the ST-EOFs in M-SSA - as suggested by Groth and Ghil (2011) - the corresponding RCs become unimodal, and the leading pair $(1,2)$ of S-EOFs in Eq. (C1) captures most of the RCs' variance.

It follows that $\mathrm{S}$-EOFs $(1,2)$ span a two-dimensional subspace in which the oscillatory mode is sufficiently well represented, and we define an instantaneous phase $\phi_{n}$ as the argument

$$
\phi_{n}=\arg \left\{u_{n, 1}+\mathrm{i} u_{n, 2}\right\}
$$

here $\left(u_{n, 1}, u_{n, 2}\right)$ are the components of the leading pair $\left\{\mathbf{u}_{1}, \mathbf{u}_{2}\right\}$ of left-singular vectors, defined as columns in $\mathbf{U}$; see also Sauer et al. (1991) and Gibson et al. (1992), who discuss PCs in the state space reconstruction.

The usefulness of Eq. (C1) in the characterization of oscillatory behavior in $\mathbf{r}$ further emerges when describing phase relations between different channels $\mathbf{r}_{d}$. Projecting $\mathbf{r}_{d}$ onto $\left\{\mathbf{u}_{1}, \mathbf{u}_{2}\right\}$ gives the spatial phase $\theta_{d}$ as the argument

$$
\theta_{d}=\arg \left\{\mathbf{r}_{d}^{\prime} \mathbf{u}_{1}+\mathrm{i} \mathbf{r}_{d}^{\prime} \mathbf{u}_{2}\right\}
$$

According to Eq. (C1), this can be simplified as

$$
\theta_{d}=\arg \left\{s_{1} v_{d, 1}+\mathrm{i} s_{2} v_{d, 2}\right\}
$$

with $\left\{s_{1}, s_{2}\right\}$ the leading pair of singular values in $\mathbf{S}$ and $\left\{v_{d, 1}, v_{d, 2}\right\}$ the $d$-th element of the corresponding rightsingular vectors.

The phase coherence of an oscillatory mode can be quantified by means of a phase-coherence index $z$,

$$
z=\frac{\left\|\sum_{d} s_{1} v_{d, 1}+\mathrm{i} s_{2} v_{d, 2}\right\|}{\sum_{d}\left\|s_{1} v_{d, 1}+\mathrm{i} s_{2} v_{d, 2}\right\|}
$$

the ratio between resultant and total length of the sum of complex-valued vectors, with $\|\cdot\|$ the $L_{2}$ norm. The in$\operatorname{dex} z$ becomes maximal $z=1$, if all phases are equal, and $z \geq 0$ otherwise. The index $z$ is related to the measure of dispersion $1-z$, which quantifies how well vectors align (e.g., Fisher 1993). Note that $\left\|s_{1} v_{d, 1}+\mathrm{i} s_{2} v_{d, 2}\right\|^{2}=$ $s_{1}^{2} v_{d, 1}^{2}+s_{2}^{2} v_{d, 2}^{2}$ is a measure of the participation of channel $\mathbf{r}_{d}$ in the variance of $s_{1}^{2}+s_{2}^{2}$, and it is this numerator in Eq. (C5) that gives greater weight in $z$ to channels with a strong participation, i.e. with a higher variance.

\section{APPENDIX D}

\section{Procrustes algorithm}

In this section, we give a brief description of the M-SSA Procrustes algorithm from Groth and Ghil (2015) and its application to the comparison of different datasets. This comparison includes here the possibility of the datasets pertaining to different geographical regions.

Suppose we have two datasets $\mathbf{x}_{1}$ and $\mathbf{x}_{2}$ of the same length $N$, but different dimension $D_{1}$ and $D_{2}$, respectively. The M-SSA algorithm then continues in calculating the SVD of the corresponding trajectory matrices $X_{1}$ and $X_{2}$, cf. Eq. (A1),

$$
\mathbf{X}_{1,2}=\eta_{1,2}^{1 / 2} \mathbf{P}_{1,2} \boldsymbol{\Sigma}_{1,2} \mathbf{E}_{1,2}^{\prime}
$$


Given that $D_{1,2} M \geq N^{\prime}$, we obtain two T-EOF matrices $\mathbf{P}_{1,2}$ that have both the same size $N^{\prime} \times N^{\prime}$.

The M-SSA Procrustes algorithm then seeks to find an orthogonal rotation $\mathbf{T}$ that provides maximal similarity between $\mathbf{P}_{2} \boldsymbol{\Sigma}_{2} \mathbf{T}$ and $\mathbf{P}_{1} \boldsymbol{\Sigma}_{1}$. An optimal solution to this target-rotation problem is given by the orthogonal Procrustes algorithm (Green 1952; Hurley and Cattell 1962; Schönemann 1966),

$$
\mathbf{T}=\mathbf{U V}^{\prime}
$$

which yields the SVD given by

$$
\boldsymbol{\Sigma}_{2} \mathrm{P}_{2}^{\prime} \mathrm{P}_{1} \boldsymbol{\Sigma}_{1}=\mathrm{USV}^{\prime} \text {. }
$$

The rescaling of the T-EOFs by the corresponding singular value $s_{k}$ is an essential modification of the original test of Allen and Robertson (1996), which was shown by Groth and Ghil (2015) to improve the accuracy of the significance test.

Beside this so-called scaled target rotation of T-EOFs in Eqs. (D2), these authors have also discussed different possibilities of a rotation of ST-EOFs. However, in their case, both datasets are of equal size and so are $E_{1}$ and $E_{2}$. Here this is not necessarily the case, as we are interested in a comparison of datasets that have different geographical extents. In contrast to a rotation of ST-EOFs, which compares spatial aspects at each grid point, a rotation of TEOFs only compares temporal aspects of the two datasets.

Still, the solution T in (D2) for the target rotation of TEOFs in $\mathbf{P}$ comes along with an equivalent rotation $\mathbf{T}$ of ST-EOFs in $\mathbf{E}$; i.e. the SVD of $\mathbf{X}_{2}$ becomes

$$
\mathbf{X}_{2}=\eta_{2}^{1 / 2} \mathbf{P}_{2} \mathbf{T T}^{\prime} \boldsymbol{\Sigma}_{2} \mathbf{T T}^{\prime} \mathbf{E}_{2}^{\prime}
$$

with $\mathbf{T T}^{\prime}=\mathbf{I}_{\eta}$ the identity matrix. Note that this also involves a similarity transform of the singular values, $\mathbf{T}^{\prime} \boldsymbol{\Sigma}_{2} \mathbf{T}$, with non-vanishing elements off the main diagonal.

To quantify the contribution of channel $d$ to the variance $s_{k}^{2}$ of the $k$ th principal component, Groth and Ghil (2011) introduced a participation index $\pi_{d k}$ for M-SSA,

$$
\pi_{d k}=s_{k}^{2} \sum_{m=1}^{M} e_{d k}^{2}(m)
$$

it is this index that we analyze in studying the effects of a rotation T of temperature ST-EOFs in Eq. (D3). In section 5 , we are in particular interested in differences $\pi_{1}-\pi_{2}$ between a rotation $\mathrm{T}_{1}$ of the temperature T-EOFs onto the T-EOFs of the SSH field and a rotation $\mathrm{T}_{2}$ onto the T-EOFs of the wind stress forcing, respectively.

In section 4 , on the other hand, we rotate the T-EOFs of TAUY onto the T-EOFs of a joint M-SSA analysis of the TAUX, SSH, and TEMP dataset, to obtain rotated STEOFs as well as corresponding RCs for the meridional component TAUY of the wind stress.

\section{References}

Alessio, H. A., 2016: Digital Signal Processing and Spectral Analysis for Scientists: Concepts and Applications. Springer, NY, 900 pp.

Allen, M. R., and A. W. Robertson, 1996: Distinguishing modulated oscillations from coloured noise in multivariate datasets. Climate Dyn., 12 (11), 775-784, doi:10.1007/s003820050142.

Allen, M. R., and L. A. Smith, 1996: Monte Carlo SSA: Detecting irregular oscillations in the presence of colored noise. J. Climate, 9, 3373-3404, doi:10.1175/15200442(1996)009<3373:MCSDIO > 2.0.CO;2.

Berloff, P., A. Hogg, and W. Dewar, 2007: The turbulent oscillator: A mechanism of low-frequency variability of the wind-driven ocean gyres. J. Phys. Oceanogr., 37 (9), doi:10.1175/JPO3118.1.

Bjerknes, J., 1964: Atlantic air-sea interaction. Adv. Geophys., 10, 1-82.

Brachet, S., F. Codron, Y. Feliks, M. Ghil, H. Le Treut, and E. Simonnet, 2012: Atmospheric circulations induced by a midlatitude SST front: A GCM study. J. Climate, 25 (6), 1847-1853, doi:10.1175/jcli-d-1100329.1.

Broomhead, D. S., and G. P. King, 1986a: Extracting qualitative dynamics from experimental data. Physica D, 20 (2-3), 217-236, doi:10.1016/0167-2789(86)90031-X.

Broomhead, D. S., and G. P. King, 1986b: On the qualitative analysis of experimental dynamical systems. Nonlinear Phenomena and Chaos, S. Sarkar, Ed., Adam Hilger, Bristol, England, 113-144.

Carton, J. A., and B. S. Giese, 2008: A reanalysis of ocean climate using Simple Ocean Data Assimilation (SODA). Mon. Wea. Rev., 136 (8), 2999-3017, doi:10.1175/2007MWR1978.1.

Chang, K.-I., M. Ghil, K. Ide, and C.-C. A. Lai, 2001: Transition to aperiodic variability in a wind-driven double-gyre circulation model. J. Phys. Oceanogr, 31 (5), 1260-1286, doi:10.1175/15200485(2001)031<1260:TTAVIA > 2.0.CO;2.

Compo, G. P., and Coauthors, 2011: The twentieth century reanalysis project. Quart. J. Roy. Meteor. Soc., 137 (654), 1-28, doi:10.1002/qj.776.

Czaja, A., and C. Frankignoul, 1999: Influence of the North Atlantic SST on the atmospheric circulation. Geophys. Res. Lett., 26 (19), 2969-2972, doi:10.1029/1999g1900613.

Czaja, A., and C. Frankignoul, 2002: Observed impact of Atlantic SST anomalies on the North Atlantic Oscillation. J. Climate, 15 (6), 606623, doi:10.1175/1520-0442(2002)015<0606:oioasa $>2.0$.co;2.

Czaja, A., A. W. Robertson, and T. Huck, 2003: The role of Atlantic ocean-atmosphere coupling in affecting North Atlantic Oscillation variability. The North Atlantic Oscillation: Climatic Significance and Environmental Impact, J. W. Hurrell, Y. Kushnir, G. Ottersen, and M. Visbeck, Eds., Vol. 134, American Geophysical Union, 147172, doi:10.1029/134GM07.

Da Costa, E. D., and A. Colin de Verdière, 2002: The 7.7-year North Atlantic Oscillation. Quart. J. Roy. Meteor. Soc., 128 (581), 797817, doi:10.1256/0035900021643692.

Deremble, B., G. Lapeyre, and M. Ghil, 2012: Atmospheric dynamics triggered by an oceanic SST front in a moist quasi-geostrophic model. J. Atmos. Sci., 69, 1617-1632, doi:10.1175/JAS-D-110288.1. 
Deser, C., and M. L. Blackmon, 1993: Surface climate variations over the North Atlantic Ocean during winter: 19001989. J. Climate, 6 (9), 1743-1753, doi:10.1175/15200442(1993)006<1743:SCVOTN $>2.0 . \mathrm{CO} ; 2$.

Dettinger, M. D., M. Ghil, and C. L. Keppenne, 1995: Interannual and interdecadal variability in United States surface-air temperatures, 1910-87. Clim. Change, 31 (1), 35-66, doi:10.1007/BF01092980.

Dewar, W. K., 2003: Nonlinear midlatitude ocean adjustment. J. Phys. Oceanogr., 33 (5), 1057-1082, doi:10.1175/15200485(2003)033<1057:nmoa >2.0.co;2.

Dijkstra, H. A., 2005: Nonlinear Physical Oceanography: A Dynamical Systems Approach to the Large Scale Ocean Circulation and El Niño. 2nd ed., Springer-Verlag, NY.

Dijkstra, H. A., and M. Ghil, 2005: Low-frequency variability of the large-scale ocean circulation: a dynamical systems approach. Rev. Geophys., 43, doi:10.1029/2002RG000122.

Feliks, Y., and M. Ghil, 1996: Mixed barotropic-baroclinic eddies growing on an eastward midlatitude jet. Geophys. Astrophys. Fluid Dyn., 82, 137-171, doi:10.1080/03091929608213633.

Feliks, Y., and M. Ghil, 1997: Stability of a front separating water masses with different stratifications. Geophys. Astrophys. Fluid Dyn., 84, 165-204, doi:10.1080/03091929708208977.

Feliks, Y., M. Ghil, and A. W. Robertson, 2010: Oscillatory climate modes in the Eastern Mediterranean and their synchronization with the North Atlantic Oscillation. J. Climate, 23 (15), 4060-4079, doi:10.1175/2010JCLI3181.1.

Feliks, Y., M. Ghil, and A. W. Robertson, 2011: The atmospheric circulation over the North Atlantic as induced by the SST field. J. Climate, 24, 522-542, doi:10.1175/2010JCLI3859.1.

Feliks, Y., M. Ghil, and E. Simonnet, 2004: Low-frequency variability in the midlatitude atmosphere induced by an oceanic thermal front. J. Atmos. Sci., 61 (9), 961-981, doi:10.1175/15200469(2004)061<0961:LVITMA > 2.0.CO;2.

Feliks, Y., M. Ghil, and E. Simonnet, 2007: Low-frequency variability in the midlatitude baroclinic atmosphere induced by an oceanic thermal front. J. Atmos. Sci., 64 (1), 97-116, doi:10.1175/jas3780.1.

Feliks, Y., A. Groth, A. W. Robertson, and M. Ghil, 2013: Oscillatory climate modes in the Indian Monsoon, North Atlantic and Tropical Pacific. J. Climate, 26, 9528-9544, doi:10.1175/JCLI-D13-00105.1.

Feliks, Y., A. W. Robertson, and M. Ghil, 2016: Interannual variability in North Atlantic weather: Data analysis and a quasigeostrophic model. J. Atmos. Sci., 73 (8), 3227-3248, doi:10.1175/jas-d-150297.1.

Fisher, N. I., 1993: Statistical Analysis of Circular Data. Cambridge University Press.

Frankignoul, C., and K. Hasselmann, 1977: Stochastic climate models. Part II: application to sea-surface temperature anomalies and thermocline variability. Tellus, 29 (4), 289-305, doi:10.1111/j.21533490.1977.tb00740.x.

Frankignoul, C., P. Müller, and E. Zorita, 1997: A simple model of the decadal response of the ocean to stochastic wind forcing. J. Phys. Oceanogr., 27 (8), 1533-1546, doi:10.1175/15200485(1997)027<1533:asmotd >2.0.co;2.
Gámiz-Fortis, S., D. Pozo-Vázquez, M. Esteban-Parra, and Y. CastroDíez, 2002: Spectral characteristics and predictability of the NAO assessed through singular spectral analysis. J. Geophys. Res.: Atmospheres (1984-2012), 107 (D23), ACL-11, doi:10.1029/2001JD001436.

Ghil, M., and R. Vautard, 1991: Interdecadal oscillations and the warming trend in global temperature time series. Nature, 350 (6316), 324327, doi:10.1038/350324a0.

Ghil, M., and Coauthors, 2002: Advanced spectral methods for climatic time series. Rev. Geophys., 40 (1), 1-41, doi:10.1029/2000RG000092.

Gibson, J. F., J. Doyne Farmer, M. Casdagli, and S. Eubank, 1992: An analytic approach to practical state space reconstruction. Physica D, 57 (1-2), 1-30, doi:10.1016/0167-2789(92)90085-2.

Giese, B. S., and S. Ray, 2011: El Niño variability in simple ocean data assimilation (SODA), 1871-2008. J. Geophys. Res.: Oceans (19782012), 116 (C2), doi:10.1029/2010JC006695.

Green, B., 1952: The orthogonal approximation of an oblique structure in factor analysis. Psychometrika, 17 (4), 429-440, doi:10.1007/BF02288918.

Grégorio, S., T. Penduff, G. Sérazin, J.-M. Molines, B. Barnier, and J. Hirschi, 2015: Intrinsic variability of the Atlantic meridional overturning circulation at interannual-to-multidecadal time scales. $J$. Phys. Oceanogr., 45 (7), 1929-1946, doi:10.1175/JPO-D-14-0163.1.

Groth, A., and M. Ghil, 2011: Multivariate singular spectrum analysis and the road to phase synchronization. Phys. Rev. E, 84, 036206, doi:10.1103/PhysRevE.84.036206.

Groth, A., and M. Ghil, 2015: Monte Carlo singular spectrum analysis (SSA) revisited: Detecting oscillator clusters in multivariate datasets. J. Climate, 28 (19), 7873-7893, doi:10.1175/JCLI-D-150100.1.

Hanrahan, J. L., S. V. Kravtsov, and P. J. Roebber, 2009: Quasi-periodic decadal cycles in levels of lakes Michigan and Huron. J. Great Lakes Res., 35 (1), 30-35, doi:10.1016/j.jglr.2008.11.004.

Hasselmann, K., 1976: Stochastic climate models part I. Theory. Tellus A, 28 (6), doi:10.1111/j.2153-3490.1976.tb00696.x.

Hurley, J. R., and R. B. Cattell, 1962: The Procrustes program: Producing direct rotation to test a hypothesized factor structure. Behav. Sci., 7 (2), 258-262, doi:10.1002/bs.3830070216.

Hurrell, J. W., 1995: Decadal trends in the North Atlantic Oscillation. Science, 269, 676-679, doi:10.1126/science.269.5224.676.

Hurrell, J. W., Y. Kushnir, G. Ottersen, and M. Visbeck, 2003: An overview of the North Atlantic Oscillation. The North Atlantic Oscillation: Climatic Significance and Environmental Impact, J. W. Hurrell, Y. Kushnir, G. Ottersen, and M. Visbeck, Eds., Vol. 134, American Geophysical Union, Washington, DC, 1-35, doi:10.1029/134GM01.

Hurrell, J. W., and H. Van Loon, 1997: Decadal variations in climate associated with the North Atlantic Oscillation. Clim. Change, 36 (34), 301-326, doi:10.1023/A:1005314315270.

Imawaki, S., H. Uchida, H. Ichikawa, M. Fukasawa, and S.i. Umatani, 2001: Satellite altimeter monitoring the Kuroshio transport south of Japan. Geophys. Res. Lett., 28 (1), 17-20, doi:10.1029/2000g1011796. 
Jajcay, N., J. Hlinka, S. Kravtsov, A. A. Tsonis, and M. Paluš, 2016: Time scales of the European surface air temperature variability: The role of the 7-8 year cycle. Geophys. Res. Lett., 43 (2), 902-909, doi:10.1002/2015GL067325.

Jamison, N., and S. Kravtsov, 2010: Decadal variations of North Atlantic sea surface temperature in observations and CMIP3 simulations. J. Climate, 23 (17), doi:10.1175/2010JCLI3598.1.

Jiang, S., F.-f. Jin, and M. Ghil, 1995: Multiple equilibria, periodic, and aperiodic solutions in a wind-driven, double-gyre, shallow-water model. J. Phys. Oceanogr, 25 (5), 764-786, doi:10.1175/15200485(1995)025<0764:MEPAAS > 2.0.CO;2.

Jones, M. S., M. Allen, T. Guymer, and M. Saunders, 1998: Correlations between altimetric sea surface height and radiometric sea surface temperature in the South Atlantic. J. Geophys. Res.: Oceans (1978-2012), 103 (C4), 8073-8087, doi:10.1029/97JC02177.

Kaplan, A., M. A. Cane, Y. Kushnir, A. C. Clement, M. B. Blumenthal, and B. Rajagopalan, 1998: Analyses of global sea surface temperature 1856-1991. J. Geophys. Res., 103 (C9), 18 567-18, doi: $10.1029 / 97 \mathrm{jc} 01736$.

Kilpatrick, T., N. Schneider, and B. Qiu, 2016: Atmospheric response to a midlatitude SST front: Alongfront winds. J. Atmos. Sci., 73, 3489-3509, doi:10.1175/jas-d-15-0312.1.

Kondrashov, D., Y. Feliks, and M. Ghil, 2005: Oscillatory modes of extended Nile River records (A.D. 622-1922). Geophys. Res. Lett., 32 (10), L10 702, doi:10.1029/2004GL022156.

Kravtsov, S. K., W. K. Dewar, M. Ghil, P. S. Berloff, and J. C. McWilliams, 2008: North Atlantic climate variability in coupled models and data. Nonlinear Processes Geophys., 15 (1), doi:10.5194/npg-15-13-2008.

Kushnir, Y., W. A. Robinson, I. Bladé, N. M. J. Hall, S. Peng, and R. Sutton, 2002: Atmospheric GCM response to extratropical SST anomalies: Synthesis and evaluation. J. Climate, 15 (16), 22332256, doi:10.1175/1520-0442(2002)015<2233:agrtes >2.0.co;2.

Lee, T., and P. Cornillon, 1995: Temporal variation of meandering intensity and domain-wide lateral oscillations of the Gulf-Stream. J. Geophys. Res.-Oceans, 100, 13,603-13,613, doi:10.1029/95jc01219.

Liu, Z., 2012: Dynamics of interdecadal climate variability: A historical perspective. J. Climate, 25 (6), 1963-1995, doi:10.1175/2011jcli3980.1.

Marshall, J., and Coauthors, 2001: North Atlantic climate variability: phenomena, impacts and mechanisms. Int. J. Climatol., 21 (15), 1863-1898, doi:10.1002/joc.693.

Moron, V., R. Vautard, and M. Ghil, 1998: Trends, interdecadal and interannual oscillations in global sea-surface temperatures. Climate Dyn., 14 (7), 545-569, doi:10.1007/s003820050241.

Mosedale, T. J., D. B. Stephenson, M. Collins, and T. C. Mills, 2006: Granger causality of coupled climate processes: Ocean feedback on the North Atlantic Oscillation. J. Climate, 19 (7), 1182-1194, doi:10.1175/JCLI3653.1.

Paluš, M., and D. Novotná, 2004: Enhanced Monte Carlo singular system analysis and detection of period 7.8 years oscillatory modes in the monthly NAO index and temperature records. Nonlinear Processes Geophys., 11 (5/6), 721-729, doi:10.5194/npg-11-721-2004.
Penduff, T., M. Juza, B. Barnier, J. Zika, W. K. Dewar, A.-M. Treguier, J.-M. Molines, and N. Audiffren, 2011: Sea level expression of intrinsic and forced ocean variabilities at interannual time scales. $J$. Climate, 24 (21), 5652-5670, doi:10.1175/jcli-d-11-00077.1.

Pierini, S., M. Ghil, and M. D. Chekroun, 2016: Exploring the pullback attractors of a low-order quasigeostrophic ocean model: The deterministic case. J. Climate, sub judice, doi:10.1175/jcli-d-15-0848.1.

Plaut, G., and R. Vautard, 1994: Spells of low-frequency oscillations and weather regimes in the Northern Hemisphere. J. Atmos. Sci., 51 (2), 210-236, doi:10.1175/15200469(1994)051<0210:SOLFOA > 2.0.CO;2.

Robertson, A. W., 1996: Interdecadal variability over the North Pacific in a multi-century climate simulation. Climate Dyn., 12 (4), 227241, doi:10.1007/BF00219498.

Robertson, A. W., 2001: Influence of ocean-atmosphere interaction on the Arctic Oscillation in two general circulation models. J. Climate, 14 (15), 3240-3254, doi:10.1175/15200442(2001)014<3240:IOOAIO > 2.0.CO; .

Rogers, J. C., 1984: The association between the North Atlantic Oscillation and the Southern Oscillation in the Northern Hemisphere. Mon. Wea. Rev., 112 (10), 1999-2015, doi:10.1175/15200493(1984)112<1999:TABTNA > 2.0.CO;2.

Sauer, T., J. A. Yorke, and M. Casdagli, 1991: Embedology. J. Stat. Phys., 65 (3-4), 579-616, doi:10.1007/BF01053745.

Schmeits, M. J., and H. A. Dijkstra, 2000: Physics of the 9-month variability in the Gulf Stream region: Combining data and dynamical systems analyses. J. Phys. Oceanogr., 30 (8), 1967-1987, doi:10.1175/1520-0485(2000)030<1967:POTMVI > 2.0.CO;2.

Schneider, N., and B. Qiu, 2015: The atmospheric response to weak sea surface temperature fronts. J. Atmos. Sci., 72, 3356-3377, doi:10.1175/jas-d-14-0212.1.

Schönemann, P., 1966: A generalized solution of the orthogonal Procrustes problem. Psychometrika, 31, 1-10, doi:10.1007/BF02289451.

Sérazin, G., T. Penduff, S. Grégorio, B. Barnier, J.-M. Molines, and L. Terray, 2015: Intrinsic variability of sea level from global ocean simulations: Spatiotemporal scales. J. Climate, 28 (10), 4279-4292, doi:10.1175/JCLI-D-14-00554.1.

Simonnet, E., and H. A. Dijkstra, 2002: Spontaneous generation of low-frequency modes of variability in the wind-driven ocean circulation. J. Phys. Oceanogr., 32 (6), doi:10.1175/15200485(2002)032<1747:sgolfm >2.0.co;2.

Simonnet, E., M. Ghil, and H. Dijkstra, 2005: Homoclinic bifurcations in the quasi-geostrophic double-gyre circulation. J. Mar. Res., 63 (5), 931-956, doi:10.1357/002224005774464210.

Simonnet, E., M. Ghil, K. Ide, R. Temam, and S. Wang, 2003a: Lowfrequency variability in shallow-water models of the wind-driven ocean circulation. Part I: Steady-state solution. J. Phys. Oceanogr, 33 (4), doi:10.1175/1520-0485(2003)33<712:lvismo > 2.0.co;2.

Simonnet, E., M. Ghil, K. Ide, R. Temam, and S. Wang, 2003b: Low-frequency variability in shallow-water models of the wind-driven ocean circulation. Part II: Time-dependent solutions. J. Phys. Oceanogr, 33 (4), doi:10.1175/15200485(2003)33<729:1vismo > 2.0.co;2. 
Small, R., and Coauthors, 2008: Air-sea interaction over ocean fronts and eddies. Dyn. Atmos. Oceans, 45 (3-4), 274-319, doi:10.1016/j.dynatmoce.2008.01.001.

Solomon, S., D. Qin, M. Manning, Z. Chen, M. Marquis, K. B. Averyt, M. Tignor, and H. L. Miller, Eds., 2007: Climate Change 2007. The Physical Science Basis: Working Group I Contribution to the Fourth Assessment Report of the Intergovernmental Panel on Climate Change. Cambridge University Press.

Speich, S., H. Dijkstra, and M. Ghil, 1995: Successive bifurcations in a shallow-water model applied to the wind-driven ocean circulation. Nonlinear Proc. Geoph., 2, 241-268, doi:10.5194/npg-2-241-1995.

Stocker, T. F., and Coauthors, Eds., 2013: Climate Change 2013. The Physical Science Basis: Contribution of Working Group I to the Fifth Assessment Report of the Intergovernmental Panel on Climate Change. Cambridge University Press.

Sushama, L., M. Ghil, and K. Ide, 2007: Spatio-temporal variability in a mid-latitude ocean basin subject to periodic wind forcing. Atmos.Ocean, 45 (4), 227-250, doi:10.3137/ao.450404.

Sutton, R. T., and M. R. Allen, 1997: Decadal predictability of North Atlantic sea surface temperature and climate. Nature, 388 (6642), 563-567.

Uppala, S. M., and Coauthors, 2005: The ERA-40 reanalysis. Quart. J. Roy. Meteor. Soc., 131 (612), 2961-3012, doi:10.1256/qj.04.176.

Vannitsem, S., J. Demaeyer, L. De Cruz, and M. Ghil, 2015: Lowfrequency variability and heat transport in a low-order nonlinear coupled ocean-atmosphere model. Physica D, 309, 71-85, doi:10.1016/j.physd.2015.07.006.

Vautard, R., and M. Ghil, 1989: Singular spectrum analysis in nonlinear dynamics, with applications to paleoclimatic time series. Physica $D$, 35 (3), 395-424, doi:10.1016/0167-2789(89)90077-8.

Visbeck, M., E. P. Chassignet, R. G. Curry, T. L. Delworth, R. R. Dickson, and G. Krahmann, 2003: The ocean's response to North Atlantic oscillation variability. The North Atlantic Oscillation: Climatic Significance and Environmental Impact, J. W. Hurrell, Y. Kushnir, G. Ottersen, and M. Visbeck, Eds., Vol. 134, American Geophysical Union, Washington, DC, 113-145, doi:10.1029/134GM06. 